\section{The association between hypoxia, chronic ischemia and alters prostate structure and progress of chronic prostatic disease}

\author{
Mauro Luisetto ${ }^{1 *}$, Behzad Nili Ahmadabadi², Ghulam Rasool \\ Mashori ${ }^{3}$ and Gamal Abdul Hamid ${ }^{4}$
}

${ }^{1}$ European Specialist, Lab Medicine, Branch General Toxicology, Pharmacy and Pharmacology, IMA Academy, Italy

2Innovative Pharmaceutical Product Development Specialist, USA

${ }^{3}$ Department of Medical \& Health Sciences for Woman, Peoples University of Medical and Health Sciences for Women, Pakistan

${ }^{4}$ Hematology and Oncology, University of Aden, Yemen

\section{Abstract}

Chronic prostatitis today show high level of relapses and recurrent pathological events even if using the best pharmacological therapy. A better understanding of physiopathological effect of ischemic hypoxic condition (pelvic, prostate tissue) and the lymphatic congestion in same body region contribute in evolution of a complex condition. The same focusing the strategy in biofilm reduction or in leukocyte infiltration can be a right way to reduce relapses and progression of the prostatic disease. Hypoxia is also related to prostatic cancer progression and prostatic biofilm if responsible of making a new micro- environment often drug resistance. A deep knowledge in this kind of phenomena can improve the clinical effect of drug therapy.

\section{More Information}

*Address for Correspondence: Mauro

Luisetto, European Specialist Lab Medicine, Branch General Toxicology, Pharmacy and Pharmacology, IMA Academy, Italy,

Email: maurolu65@gmail.com; m.luisetto@ausl.pc.it

Submitted: 29 August 2019

Approved: 19 September 2019

Published: 20 September 2019

How to cite this article: Luisetto $M$, Ahmadabadi BN, Mashori GR, Hamid GA. The association between hypoxia, chronic ischemia and alters prostate structure and progress of chronic prostatic disease. Arch Pharm Pharma Sci. 2019; 3: 042-078.

DOI: dx.doi.org/10.29328/journal.apps.1001016

Copyright: (C) 2019 Luisetto M, et al. This is an open access article distributed under the Creative Commons Attribution License, which permits unrestricted use, distribution, and reproduction in any medium, provided the original work is properly cited

Keywords: Chronic prostatitis; Ischemia; Hypoxia; Physiopathology; Antimicrobials; Prostatic-pelvic congestion; Prostate kinetics; Prostate cancer progression

Check for updates

\section{Introduction}

In order to start this work is interesting to observe some factors involved in progression of chronic prostatitis like antibiotic resistances, biofilm, pharmakokinetics of drugs into prostate tissue, local hypoxia, flogosys, leucocyte infiltration, micro calcifications, lifestyle behavior, associated comorbidities and many other.

According article: Excessive Antibiotic Use in Men with Prostatitis by Brent C Taylor, et al: "Antibiotic overuse has been identified as 1 of 20 priority areas for improving health care quality. 20 Increased antibiotic use contributes to the development and spread of antibiotic-resistant bacteria. Once confined to the inpatient setting, resistant bacteria are now common community acquired infections. The increasing prevalence of antibiotic resistant organisms is associated with morbidity and mortality and has led to more expensive and broad-spectrum empiric antibiotic prescribing.

Despite evidence that antibiotics are not effective in the majority of men with chronic pelvic pain syndrome, they were prescribed in $69 \%$ of men with this diagnosis even after excluding men diagnosed with infectious/acute prostatitis or urinary tract infections. Some increased use is possibly the result of uncontrolled confounding by coexisting conditions or misclassification. The multivariable-adjusted 7-fold higher rate of fluoroquinolone use is likely the result of excessive prescribing in a population unlikely to benefit from treatment. Quality improvement strategies to reduce unnecessary antibiotic use in men with chronic prostatitis are warranted" 2008.

And as reported in article: Chronic Prostatitis: Approaches for Best Management by Kyung Seop Lee, et al: "The optimal management of category III prostatitis is not known. Conventional prolonged courses of antibiotic therapy have not proven to be efficacious" 2012. Is clear that actually pharmacological therapy of chronic prostatitis and related condition is characterized by high antibiotics abuse.

Some strategies can help to reduce this and one of this is to correlate the global amount of antibiotic use in a periods 
(1-3-6 month) to behavior lifestyle modification and with an anti-biofilm strategy. This works start from the observation if in animal world Mammalia, primates, humans excluded, there is the same ratio of chronic prostatic condition or not and what can be the real reasons. The same is interesting to observe in example if dogs or cats or other animals use lots of antibiotics to treat lower urinary tract infectious disease like humans today.

Obviously there are relevant differentials. Also evolutionary process and actual in natural sitting position of humans contribute in a general artificial pelvic congestion like situation. In this condition also the evolutionary bipedal position change from quadruped of primates produced different kind of bladder and prostatic empty. Chair and seat often are very rigid in working setting (traumatic damage), and some sports like bicycle often produce micro trauma in prostate region (8 hours in a sitting condition added in an obese man can produce an dangerous Condition for a delicate organ like prostate tissue).

From literature is possible to verify that hypoxic condition, flogosys, bacteria, ageing (IPB), fibrotic reaction, lymphocyte, micro calcifications, biofilms and other Factor can exacerbate relapse of chronic prostatitis and related conditions.

And According the Mayo clinic about prostatitis treatments:

https://www.mayoclinic.org/diseases-conditions/prostatitis/ diagnosis-treatment/drc-20355771 Lifestyle and home remedies:

"The following might ease some symptoms of prostatitis: Soak in a warm bath (sitz bath) or use a heating pad, Limit or avoid alcohol, caffeine, and spicy or acidic foods, which can irritate your bladder. Avoid activities that can irritate your prostate, such as prolonged sitting or bicycling, drink plenty of caffeine-free beverages. This will cause you to urinate more and help flush bacteria from your bladder". So Innatural posture, prolonged sitting position, linphatic congestion, venous circle stasis. Prostate kinetics, Flogotic reactions contribute to produce Fibrotic reaction. Body weight, Ageing, bacteria are all factors that in cumulative way increase the pathological movement in prostatitis.

According article: "Receptor pharmacology and other relevant factors in lower urinary tract pathology under a functional and toxicological approach: Instrument to better manage antimicrobials therapy". Arch Pathol Clin Res. 2018:

"In various patients conditions involved in lower urinary tract disease LUT (like overactive bladder, bladder neck sclerosis, dis-synergy (without synenrgic contraction between bladder detrusor and bladder neck, BPH, recurrent cysytitis, interstitial cystitis, chronic prostatitis, uretral stenosis, loss of sphinteric coordination, Prostatic cancer, anatomic abnormalities and other factors the receptor status play relevant role to reduce effect of vicious circle that can be responsible in progression of the pathologic process.
In Various prostatic, bladder neck or urethral condition a reduced urinary fluss can produce infectious conditions like acute or chronic prostatitis.

Irritants substantives in diet (in example ethylic alcohol drink, hot spices, crud meats, carbonate drinks, caffeine and other) can produce Painful stimulus in innervations of vesicle trigonous, bladder neck and prostatic urethra. The same recurrent cystitis and $\mathrm{BPH}$ contribute in a complex situation. This stimulus produce hypertonus of bladder muscle involved in the expulsion of urine. The event related inflammation and edema (bladder, prostatic urethra, trigonus) contribute to the global effect. So conditions like bladder neck sclerosis IPB, recurrent prostatitis and cystitis in acts in a vicious circle (Also immunomediated: Bph and cronic prostatitis with lymphocytic infiltration and tissue remodeling).

The hormonal status check the systems (see 5-ARI efficacy in BPH). Sympathetic, parasympathetic and other system are deeply involved. Also behavioral habits or diet can influence in example urinary flux in a complex system like LUT. (Bladder and prostatic irritants that can produce edema and acute inflammation). Other behavior habits are deeply involved as too much sedentary, water intake, coffee and also psychological profile le and stressing conditions.

Some disease like diabetes produce high consequences in all this systems due to Bladder modification, oxidative stress, osmotic movens, and increase susceptibility of urinary infections. The anatomic abnormalities produces, obviously, physiological dysfunctions.

Recurrent urinary tract infections, inadequate antimicrobial therapy:

Profile of resistance, duration of therapy, kind of antimicrobials, posology, Pk. Kinetics, associations, compliance, biofilms, micro calcifications (recurrent chronic prostatitis) contribute to a progression of the condition".

\section{Material and Methods}

With an observational approach some relevant (in our opinion) biomedical literature (PUBMED) is analyzed to produce a new global conclusion. The literature funded is related congestive pelvic and prostatic condition, life style, ANTI- biofilms strategies and other relevant for the scope of this work. High relevance is reserved to recent anti - biofilm strategy under research for some biofilm relate human disease. An experimental project hypotesys is then submitted to the researcher to better explain the concept.

\section{Results}

\section{Form literature}

Kogan, et al: "To examine the structure of the prostate tissue in patients with III B chronic prostatitis (CP) and 
chronic pelvic pain syndrome (CPPS). The study analyzed transrectal fine-needle biopsy specimens of 10 patients with the verified diagnosis of chronic pelvic pain syndrome/ category III B chronic prostatitis (CPPS/IIIB CP) according to the National Institutes of Health classification. Tissues were examined using light and electron microscopy, and immunohistochemical study of the expression of CD31, CD34, NSE and S-100 markers.

All biopsy specimens of all patients showed fibroplastic changes of the prostate tissue most markedly pronounced in the stroma and muscle fibers in the form of total fibrosis, myofibril atrophy, and extracellular lipofuscin deposition. The examination revealed a significant reduction in the density of microcirculatory bed vessels and arteriolar luminal stenosis, a reduction in the number of nerve fibers, and compression of their fibrous tissue. No inflammatory changes were found in the prostate.

In patients with CPPS/IIIB CP, the changes in the prostate at the microscopic and ultra-structural levels are characteristic of severe chronic tissue hypoxia, which leads to the development of fibrosis resulting in stenosis of microcirculatory bed vessels and degenerative changes in nerve fibers and cells. No signs of an inflammatory reaction in the examined tissue were established. Changes in the prostate tissue in CPPS/IIIB CP suggest the presence of chronic pelvic ischemia and exclude its association with inflammation as the main pathological process" [1].

Ram Vaidyanathan, et al: "A myriad of etiological factors - some not even involving the prostate gland - have been postulated. The initiator of the inflammatory process could be a local infection, chemical irritation, dysfunctional voiding, intraductal reflux, neuromuscular disturbances or an immunological process. There may be an etiological link between Category III prostatitis and interstitial cystitis (IC). The pathogenesis is not entirely certain, but the following may be a possible mechanism. Regardless of the triggering factor, the resultant inflammatory process causes tissue edema and increased intra-prostatic pressure leading to local hypoxia and varied mediator-induced tissue damage. This leads to altered neurotransmission in sensory nerve fibers thereby resulting in the pain and other symptoms associated with the condition" [2].

Simon Allen, et al: "In recent years, researchers have suggested that the pathogenesis of BPH may be associated with vascular dysfunction, prostatic hypoxia, pelvic ischemia and elevated pressure in the prostate gland" [3].

Kogan MI, et al: "Causes and mechanisms of chronic pelvic pain/chronic prostatitis (CPP/CP) syndrome are not well investigated yet. It is suggested that pain in an inflammatory form of this syndrome may be due to tissue inflammation. In the non-inflammatory form pain may result from neurogenic factors. We made duplex mapping of the prostate and obtained quantitative characteristics of arterial hemodynamics of the prostate. Control values of arterial prostatic blood flow were developed after examination of healthy volunteers. These values were compared with similar values of arterial blood flow in CPP/CP syndrome. We found a significant decrease of the peak systolic velocity in patients with non-inflammatory CPP/CP syndrome. There is a direct correlation between intensity of pain syndrome and peak systolic velocity of arterial blood flow. Thus, pain may arise from chronic prostatic ischemia" [4].

Kim HJ, et al: "Benign prostatic hyperplasia (BPH) commonly occurs in older men with chronic prostatitis. Although BPH is frequently accompanied by inflammation, it is unclear whether inflammation underlies prostate enlargement. Recently, we reported that hypoxia-inducible factor $1 \alpha$ (HIF-1 $\alpha)$, which is known to be induced by proinflammatory cytokines, is involved in testosteroneinduced prostate hyperplasia. We hypothesized that cytokines secreted from infiltrated macrophages under inflammatory conditions stimulate prostate enlargement by up-regulating HIF-1 $\alpha$. In the present study, we injected lipopolysaccharide (LPS) into rat prostates to mimic prostatitis and evaluated prostate hyperplasia 14days later. Epithelial cells of LPS-treated prostates were found to be highly proliferative and HIF- $1 \alpha$ levels in prostate tissues to be elevated. When prostate epithelial cells were incubated in conditioned medium from macrophages activated with LPS, they robustly expressed HIF- $1 \alpha$, and under these conditions IL- $1 \beta$, IL- 6 , and TNF- $\alpha$ cytokines were found to mediate HIF$1 \alpha$ induction. HIF-1 $\alpha$ was found to enhance the expression of Twist, which initiates epithelial-mesenchymal transition (EMT). Profound EMT features were observed in LPS-treated rat prostates, and the natural HIF- $1 \alpha$ inhibitors ascorbate and curcumin were found to attenuate EMT and prostate hyperplasia both in vivo and in vitro. Based on these results, we propose that HIF- $1 \alpha$ mediates prostate enlargement under inflammatory conditions, and we suggest that HIF- $1 \alpha$ be viewed as a promising target for blocking the transition from prostatitis to $\mathrm{BPH}$ " [5].

Portia Thurmond, et al: "Clinical studies have reported a correlation between pelvic ischemia and voiding dysfunction in elderly men. The aim of this study was to identify and compare prostate structural modifications in cultured cells and in a rabbit model after exposure to hypoxia, oxidative stress, and chronic ischemia. Cultured human prostate smooth muscle cells (SMCs), epithelial cells (ECs), and stromal cells (SCs) were incubated under normoxia, hypoxia, and oxidative stress conditions by use of a computerized oxycycler system. We developed a rabbit model of chronic prostate ischemia by creating aorto-iliac arterial atherosclerosis. Markers of oxidative stress were examined by using fluorometric analysis and enzyme immunoassay. Prostate structure was examined by using Masson's trichrome staining and transmission electron microscopy (TEM). 
Lipid peroxidation was found in SMCs exposed to hypoxia and in all cell types exposed to oxidative stress. We identified protein oxidation in ECs exposed to hypoxia and in all cell types exposed to oxidative stress. Markers indicating oxidative damage were present in chronically ischemic rabbit prostate tissue. These reactions were associated with DNA damage. Prostate ischemia resulted in epithelial atrophy, loss of smooth muscle, and diffuse fibrosis. TEM showed swollen mitochondria with degraded cristae, loss of membrane, loss of Golgi bodies, degenerated nerves, and disrupted cell-tocell junctions.

Human prostate cells exhibited differential reactions to hypoxia and oxidative stress with widespread DNA damage. Structural modifications in ischemic prostate tissue were similar to those in cells exposed to oxidative stress. Structural changes due to ischemia and oxidative stress may contribute to prostatic noncompliance in aging men.

\section{Differential reactions of human prostate cells to hypoxia and oxidative stress}

Protein oxidation, characterized by increased AOPP levels, was detected in ECs exposed to hypoxia and in SMCs, ECs, and SCs exposed to oxidative stress (Figure 1). Lipid peroxidation, characterized by MDA upregulation, was found in SMCs exposed to hypoxia and in SMCs, ECs, and SCs exposed to oxidative stress.

\section{DNA damage in human prostate cells}

Protein oxidation and lipid peroxidation in prostatic cells were associated with widespread DNA damage. SMCs, ECs, and SCs exposed to both hypoxia and oxidative stress demonstrated significantly increased 8-0HdG levels, suggesting DNA damage.

\section{Ultrastructural changes in human prostate SMCs}

Hypoxia impaired cell membrane structure, caused a partial loss of the outer mitochondrial membrane, and led to swollen, enlarged endoplasmic- reticulum (ER) and glycogen accumulation. Oxidative stress produced similar changes in a more pronounced manner. SMCs exposed to oxidative stress exhibited deformed cell membranes, partial loss of mitochondrial membrane, degradation of mitochondrial cristae, enlarged splintered ER, and accumulation of cytoplasmic lysosomes.
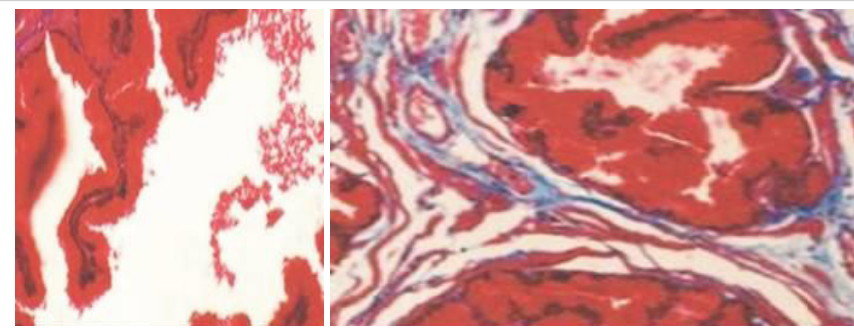

Figure 1: Masson's trichrome staining of rabbit ischemic prostate tissues $(\times 40)$ showed stromal thickening with diffuse fibrosis, decreased smooth muscle fibers, distorted glands with flattened epithelium lining, and large intraluminal spaces compared to prostatic tissues from the sham group $(\times 40)$.[6].
Masson's trichrome staining of rabbit ischemic prostate tissues $(\times 40)$ showed stromal thickening with diffuse fibrosis, decreased smooth muscle fibers, distorted glands with flattened epithelium lining, and large intraluminal spaces compared to prostatic tissues from the sham group $(\times 40) "[6]$.

Kimio Sugaya, et al: "Pathological inflammatory changes and glandular atrophy of the prostate were more severe in the castration combined with pelvic congestion and castration combined with pelvic congestion plus eviprostat groups than in the sham group" [7] (Figure 2).

Coker TJ, et al: "Most cases of acute bacterial prostatitis are caused by ascending urethral infection or intraprostatic reflux and are facilitated by numerous risk factors. These infections may occur from direct inoculation after transrectal prostate biopsy and transurethral manipulations (catheterization and cystoscopy). Occasionally, direct or lymphatic spread from the rectum or hematogenous spread via bacterial sepsis can cause acute bacterial prostatitis. Community-acquired infections are three times more common than nosocomial infections" [8].

Shazia R Chaudhry, et al: "Blood Supply and Lymphatics at the pelvic inlet, the common iliac artery divides into internal and external iliac arteries. The internal iliac artery provides oxygenated blood to pelvic viscera including bladder, prostate in males, urethra, uterus in the female, and vagina in females. Branches of internal iliac artery include the superior vesical artery, obturator artery, inferior vesical artery, uterine artery, middle rectal artery, internal pudendal artery, and inferior gluteal artery. Venous drainage is achieved primarily by the internal iliac vein. The internal iliac vein combines superiorly with the external iliac vein to form the common iliac -vein which then drains into the inferior vena cava" [9].

Chiappino G, et al: "Prostatitis is a poorly defined group of syndromes with multiple causes. Chronic prostatitis may be non-bacterial and due to intra-pelvic venous congestion. If the causes persist and adequate treatment is not given, the congestive syndrome may become chronic and interfere with fertility with severe biological damage. Little is known in the field of occupational medicine (as regards clinical and
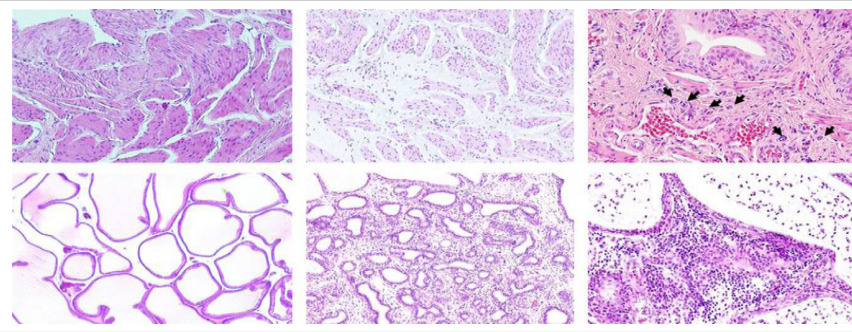

Figure 2: (d) Mild edema in the stroma of the prostate in a rat from the sham group (magnification: 9100). (e) Severe glandular atrophy and stromal fibrosis of the prostate in a rat from the PC-C/Evi group (magnification: 9100). (f) Infiltration of inflammatory cells (mainly lymphocytes) into the stroma, and infiltration of inflammatory cells (mainly lymphocytes and neutrophils) into the glandular cavities of the prostate in a rat from the PC-C/Evi group (magnification: 9200) [7]. 
pathogenic aspects) and, on the other hand, little is known by urologists (as far as the aetiological aspects are concerned), the prostatitis-like syndrome due to intrapelvic congestion has been defined in recent studies as non-bacterial prostatitis or prostatodynia, but we prefer to call it "prostatosis". The results of a close cooperation between urologists and occupational physicians are reported. Patients with nonbacterial chronic prostatosis were evaluated from the urological and occupational point of view and all the etiological factors of both occupational and no occupational origin were considered. When occupational factors are a condition sine qua non prostatosis is considered an occupational disease.

Two cases of "occupational prostatosis" are described where driving vehicles and a sedentary employment played the most important etiological part. Close co-operation between urologists and occupational physicians makes it possible to complete clinical diagnosis with a careful evaluation of all the factors of both occupational and non-occupational origin and allows the identification of those cases that must be defined as occupational diseases. Prostatosis due to venous congestion deserves the attention of occupational physicians since the distinction between occupational and non-occupational origin must be found both in individual cases and in groups of workers subject to the same factors. The present state of knowledge is enough to take preventive measures aimed at reducing the frequency of new cases and avoiding the deterioration of existing cases. It is also possible that some cases of pseudo-cystitis in women might be the result of intrapelvic venous congestion of occupational origin. Anatomical and physiological nonoccupational factors as well as certain habits of life style can favor intrapelvic venous congestion, producing conditions of hypersusceptibility to occupational factors and could sometimes cause the disease even in the absence of causes connected to work" [10].

Konstantinos Stamatiou, et al: "In total, 188 out of 314 Gram-positive bacterial isolates were monomicrobial and the remaining 126 polymicrobial. A vast variety of Gram-positive bacteria was found in positive cultures, with coagulase negative Staphylococci (CoNS, mainly S. haemoliticus, S. hominis, S. epidermidis and rarely $\mathrm{S}$. lugdunensis) being the most frequent pathogens (85monomicrobial and 43 polymicrobial isolates). As far as the outcomes of followup visits are concerned, bacterial eradication was achieved in 213 cases though 135 were completely clinically cured. In the remaining 78 cases bacterial elimination was not accompanied by clinical improvement. Bacterial persistence occurred in 70 cases. 41 out of these were super infections and the remaining 29 were true persistences. The data from the present study suggest that Gram-positive pathogens can be responsible for prostatic infection. Multi-drug resistance for CoNS and Enterococci is an emerging medical problem that may cause important threats to public health in the future. In this study, tetracyclines and macrolides were successfully demonstrated to be an alternative to quinolones.
The pathogens most commonly associated with both clinical relapses and super infections were Enterococcus faecalis, and CoNS. To our knowledge, Gram+ cocci like Enterococcus faecalis are at the same time the most common uropathogens and the bacteria carrying the most powerful resistance determinant. Emerging molecular data and special culture results suggest that CoNS species cause bacterial prostatitis relapses while both Enterococcus faecalis and CoNSare biofilm formators. The data from the present study suggest that Gram-positive bacteria do colonize the urethra and/or prostatic ducts, and can be responsible forprostatic infection. Multi-drug resistance in CoNS and Enterococci is an emerging medical problem that may cause important threats to public health in the future" [11].

Azadzoi KM, et al: "They studied the effect of chronic ischemia on prostatic smooth muscle contraction in the rabbit. New Zealand male rabbits weighing 3 to $3.5 \mathrm{~kg}$ were assigned to 2 groups. Group 1 ( 10 rabbits) underwent balloon endothelial injury of the iliac arteries and received a $0.5 \%$ cholesterol diet for 4 weeks and then a regular diet for 8 weeks. Control group 2 ( 10 rabbits) received a regular diet. After 12 weeks the animals were anesthetized. Iliac artery and prostate blood flow was recorded. Prostate tissues were prepared for isometric tension measurement, enzyme immunoassay to determine cyclic guanosine monophosphate (cGMP) release and histological examination. In group 1 atherosclerosis as well as a significant decrease in iliac artery and prostate blood flow were observed. Ischemia significantly increased prostatic tissue contraction, decreased cGMP release and led to capsular and stromal thickening, and epithelial atrophy. The alpha1-adrenoceptor blocker doxazosin and the phosphodiesterase- 5 inhibitor sildenafil citrate significantly decreased the contraction of control and ischemic tissues. Doxazosin was more effective in decreasing contractions when it was combined with sildenafil or the nitric oxide (NO) precursor L-arginine. In contrast, doxazosin was less effective when it was combined with the NO synthase inhibitor $\mathrm{N}$ omega-nitro-L-arginine or with the guanylate cyclase inhibitor methylene blue. Doxazosin significantly increased cGMP release in control tissues but not in ischemic tissues. Sildenafil significantly increased cGMP release in control and ischemic tissues. Ischemia increased prostatic smooth muscle contraction and led to marked structural damage. Stimulators of NO synthesis and cGMP production enhanced the efficacy of doxazosin in decreasing prostatic tissue contraction. Sildenafil decreased contractility and increased cGMP release. Increased smooth muscle tone and structural changes in the ischemic prostate may suggest a role for prostate ischemia in resistance to urinary flow independent of prostate size" [12].

Berger AP, et al: "To assess benign prostatic hyperplasia (BPH) and erectile dysfunction (ED), both considered to be associated with urogenital ageing, in ageing men in a crosssectional population study, comparing them with healthy controls by using symptom scores and contrast-enhanced 
colour Doppler ultrasonography (CDUS). Trans-rectal CDUS and quantitative measurement of colour pixel intensity (CPI) are excellent minimally invasive techniques for assessing normal and pathological blood flow. CDUS was performed using the microbubble-based ultrasound enhancer for evaluating prostate, bladder neck and corpus cavernosum vascularity in young healthy men, men with $\mathrm{BPH}$, and men with severe vascular damage (diabetes mellitus type 2). Resistive index measurements and computer-assisted quantification of CPI were used to objectively evaluate perfusion. The International Prostate Symptom Score (IPSS) and the International Index of Erectile Function (IIEF) were applied to quantify the symptoms. In patients with $\mathrm{BPH}$, perfusion of the transition zone (TZ) of the prostate was significantly lower and the resistive index of the TZ significantly higher (both $P<0.001$ ) than in healthy controls. The perfusion patterns of men with BPH and those who also had severe vascular damage (diabetes mellitus type 2) showed that vascularity in the latter group was lower in the prostatic TZ and the corpora cavernosa. In patients with BPH the IPSS, quality-of-life and IIEF scores were significantly worse than in the control group. Men with concomitant atherosclerosis had even worse symptom scores. These results strongly support the hypothesis that age-related impairment of blood supply to the lower urinary tract is important in the development of BPH and ED. Vascular damage may cause chronic ischaemia and thus be a contributing factor in the pathogenesis of BPH and ED" [13].

Chen $\mathrm{X}$, et al: "Chronic prostatitis/chronic pelvic pain syndrome (CP/CPPS) is a common problem with unclear etiology. Some diet and lifestyle factors were thought to correlate with CP/CPPS, but studies comprehensively investigate this correlation are rarely available. The current study was conducted to determine the potential lifestylerelated risk factors of $\mathrm{CP} / \mathrm{CPPS}$ and its pain severity in Chinese population. Participants were recruited from seven hospitals in Shanghai from July 2012 to August 2013. Demographics, medical history, diet and lifestyle information, and CP/CPPS symptoms were obtained from each participant using a questionnaire. Univariate and multivariate logistic regression analyses were used to identify potential lifestylerelated risk factors for $\mathrm{CP} / \mathrm{CPPS}$ and its pain severity. A total of 784 men with CP/CPPS and 785 controls were enrolled in this study. Multivariate regression model indicated that age, nightshift work, stress, smoking status, alcohol consumption, less water intake, imbalanced diet, frequent sexual activity, delaying ejaculation and holding urine were identified as potential risk factors for $\mathrm{CP} / \mathrm{CPPS}$, whereas sedentary lifestyle, caffeinated drinks and less water intake were associated with severe pain in CP/CPPS patients. Several diet and lifestyle factors associated with CP/CPPS and pain severity were determined in this study. These modifiable conditions are potential targets for treatment of CP/CPPS. Further studies are necessary to determine their role in the pathogenesis of CP/CPPS" [14].
J Curtis Nickel, et al: "These EBM recommendations highlight the fact that the traditional biomedical model of dealing with chronic prostatitis has failed many patients. So where do we go now? The answer will lie in our evolving understanding of the pathophysiology and etiology of CP/CPPS. It is now apparent that the condition occurs in anatomically and/or genetically susceptible men who suffer from some initiator factor (usually repetitive). These initiators can be infection (urethritis, cystitis, prostatitis), dysfunctional high-pressure voiding (bladder neck, prostate, sphincter, or urethral pathology), failure to relax the pelvic floor muscles at rest or during voiding, trauma (bicycle seat, prolonged sitting), or allergic phenomenon. This can lead to a self-perpetuating immunologic inflammatory state and/ or neurogenic injury, creating acute and then chronic pain. Peripheral and then central nervous system sensitization involving neuroplasticity may lead to a centralized neuropathic pain state, further modulated by upper central nervous system centers. New avenues of therapy will involve novel diagnostic strategies leading to neuromodulatory, physical, and cognitive-behavioral therapies. Such treatment trials are already ongoing and hold promise for better management of CP/CPPS. Although most published studies have shown the benefit of physical therapy interventions, none has been controlled. Physical therapy should address most of the following:

1. Education of the patient about pelvic muscle function and pain

2. Education about lifestyle issues that may exacerbate the pain

\section{Education about how posture affects the pelvis}

4. Education about exercises that may be of benefit and those that may be harmful

\section{Specific stress-reduction techniques}

6. Manual therapy such as myofascial trigger-point release and joint mobilization

7. Specific exercises to improve strength, relax muscles, and restore balance

8. Exercise aimed at improving core posture and general health and well-being

9. Education about voiding and sexual behaviors that may exacerbate the problem" [15].

Gao DJ, et al: "To investigate the prevalence and related factors of prostatitis-like symptoms among young men. The study was a cross-sectional survey of 2500 young men aged 18-30 years in the city of Weifang, and all of them completed a questionnaire on prostatitis. The univariate and multivariate logistic regression procedures were used to investigate the 
risk factors among the young men with chronic prostatitislike symptoms. The valid response rate was $85 \%(\mathrm{n}=2125)$. Of the 128 subjects (6.02\%) identified as having chronic prostatitis-like symptoms, the mean age was 21.8 years, the average pain score was $6.98+/-0.29$, and the average voiding score was $3.77+/-0.25$. Of the sampled population, 39 men had prostatitis-like symptoms with an index pain score of 8 or more. Significant risk factors include frequent masturbation, prolonged sitting, long-time fixed posture, cold environment, stress at home and work. The study suggested that chronic prostatitis-like symptoms are common among young men, and the urethritis history, frequent masturbation, prolonged sitting, long-time urine holding, cold environment, and stress at home and work might be significant risk factors" [16].

Brent C Taylor, et al: "Prostatitis accounts for two million outpatient visits annually. The vast majority of prostatitis cases fit the definition of chronic pelvic pain syndrome for which routine antibiotic use is not indicated. Inpatient, outpatient, and pharmacy datasets from the veterans' health administration were used to quantify the magnitude of antibiotic use attributable to chronic pelvic pain syndrome. Specifically, men with a diagnosis of infectious/acute prostatitis, and/or a urinary tract infection were excluded, and the remaining men with a diagnosis of prostatitis were defined as having chronic pelvic pain syndrome.

Annual prevalence of chronic pelvic pain syndrome was $0.5 \%$. Prescriptions for fluoro-quinolone antibiotics were filled in $49 \%$ of men with a diagnosis of chronic pelvic pain syndrome compared to five percent in men without chronic pelvic pain syndrome. Men with chronic pelvic pain syndrome were greater than seven times more likely to receive a fluoroquinolone prescription independent of age, race/ethnicity and comorbid conditions. Increased use of other antibiotics was also observed. High utilization was similar in men with either infectious/acute prostatitis or chronic pelvic pain syndrome.

Despite evidence that antibiotics are not effective in the large majority of men with chronic pelvic pain syndrome, they were prescribed in $69 \%$ of men with this diagnosis. Some increased use is probably due to uncontrolled confounding by comorbid conditions or inaccurate diagnostic coding. A seven-fold higher rate of fluoro-quinolone usage suggests strategies to reduce unnecessary antibiotic use in men with prostatitis are warranted.

Despite evidence that antibiotics are not effective in the majority of men with chronic pelvic pain syndrome, they were prescribed in $69 \%$ of men with this diagnosis even after excluding men diagnosed with infectious/acute prostatitis or urinary tract infections. Some increased use is possibly due to uncontrolled confounding by coexisting conditions or misclassification. The multivariable-adjusted seven-fold higher rate of fluoro-quinolone use is likely due to excessive prescribing in a population unlikely to benefit from treatment. Quality improvement strategies to reduce unnecessary antibiotic use in men with chronic prostatitis are warranted" [17].

Jinjin Ma, et al: “Current therapies for male lower urinary tract symptoms secondary to prostate enlargement prevent hormonal effects on prostate growth and inhibit smooth muscle contraction to ease bladderneckand urethral pressure. Lower urinary tract symptoms can be refractory to these therapies, suggesting that additional biological processes not addressed by them may also contribute to lower urinary tract symptoms. Aging associated fibrotic changes in tissue architecture contribute to dysfunction in multiple organ systems. Thus, we tested whether such changes potentially have a role in impaired urethral function and perhaps in male lower urinary tract symptoms. Periurethral tissues were obtained from a whole prostate ex vivo and from 28 consecutive men treated with radical prostatectomy. Lower urinary tract symptoms were assessed using the American Urological Association symptom index. Prostate tissues were subjected to mechanical testing to assess rigidity and stiffness. Fixed sections of these tissues were evaluated for collagen and elastin content, and glandularity to assess fibrosis. Statistical analysis included the Student t test and calculation of Pearson correlation coefficients to compare groups.

Periurethral prostate tissues demonstrated nonlinear viscoelastic mechanical behavior. Tissue from men with lower urinary tract symptoms was significantly stiffer $(p=0.0016)$ with significantly higher collagen content ( $p=0.0038)$ and lower glandularity than that from men without lower urinary tract symptoms (American Urological Association symptom index 8 or greater vs 7 or less).

Findings show that extracellular matrix deposition and fibrosis characterize the periurethral prostate tissue of some men with lower urinary tract symptoms. They point to fibrosis as a factor contributing to lower Urinary tract symptom etiology" [18] (Figure 3).

Carsten Niemitz:"During the last century, more than 30 hypotheses have been constructed to explain the evolution
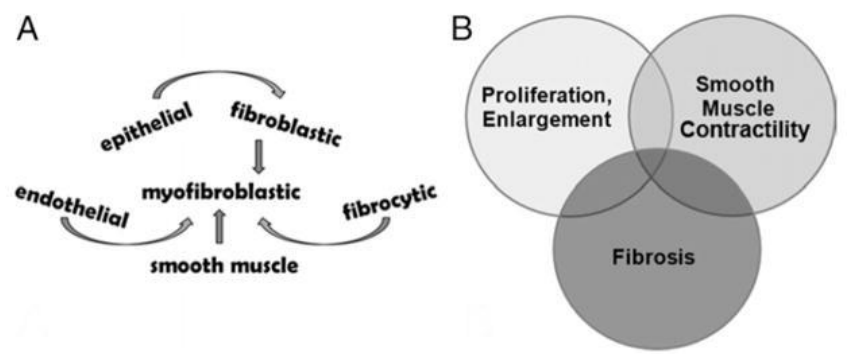

Figure 3: Prostate fibrotic development and contribution to male LUTS. A, multiple cell types that can differentiate into myofibroblastic cells. B, 3 major pathobiological processes that can act alone or in combination to promote male LUTS [18]. 
of the human upright posture and locomotion. It has been established that all main hypotheses published until the last decade of the past century are outdated, at least with respect to some of their main ideas: Firstly, they were focused on only one cause for the evolution of bipedality, whereas the evolutionary process was much more complex. Second, they were all placed into asavannah scenario. During the 1990s, the fossil record allowed the reconstruction of emerging bipedalism more precisely in a forested habitat. The fossil remains revealed increasing evidence that this part of human evolution took place in a more humid environment than previously assumed. The Amphibian Generalist Theory, presented first in the year 2000, suggests that bipedalism began in a wooded habitat. The forests were not far from a shore, where our early ancestor, along with its arboreal habits, walked and waded in shallow water finding rich food with little investment. In contrast to all other theories, wading behavior not only triggers an upright posture, but also forces the individual to maintain this position and to walk bipedally. Sofar, this is the only scenario suitable to overcome the considerable anatomical and functional threshold from quadrupedalism to bipedalism. This is consistent with paleoanthropological findings and with functional anatomy as well as with energetic calculations, and not least, with evolutionary psychology. The new synthesis present edhere is able to harmonise many of the hitherto competing theories" [19].

Ran Zhang, et al: "Chronic prostatitis/chronic pelvic pain syndrome (CP/CPPS) is a prevalent urologic disorder among men, but its etiology is still poorly understood. Our objective was to examine the relationship between physical activity and incidence of CP/CPPS in a large cohort of male health professionals.

We conducted a prospective cohort study among men in the Health Professionals Follow-up Study followed from 1986 to 2008 . The study population included 20,918 men who completed all CP/CPPS questions on the 2008 questionnaire. Leisure-time physical activity, including type and intensity of activity, was measured by questionnaire in 1986. A National Institute of Health Chronic Prostatitis Symptom Index pain score was calculated based on the responses on the 2008 questionnaire. Participants with pain scores $\geq 8$ were considered CP/CPPS cases $(n=689)$.

Higher leisure-time physical activity was associated with lower risk of CP/CPPS. The multivariable-adjusted odds ratio (OR) comparing $>35.0$ to $\leq 3.5 \mathrm{MET}-\mathrm{h} / \mathrm{wk}$ of physical activity was 0.72 (95\% confidence interval (CI): $0.56,0.92, p$ for trend $<0.001$ ). Observed inverse associations between physical activity and CP/CPPS were similar for both moderate- and vigorous-intensity activities. Sedentary behavior, measured as time spent watching television, was not associated with risk of CP/CPPS ( $\mathrm{p}$ for trend 0.64).

Findings from this study, the first large scale and most comprehensive study to date on this association, suggest that higher levels of leisure-time physical activity may lower risk of CP/CPPS in middle-aged and older men. Total leisuretime physical activity was significantly inversely associated with risk of CP/CPPS. Comparing > 35.0 to $0-3.5$ MET-h/ wk of physical activity, the OR for CP/CPPS was 0.72 (95\% CI $0.56-0.92$, $\mathrm{p}$ for trend $<0.001$ ). When associations between leisure-time physical activity and CP/CPPS were examined separately by intensity, inverse associations were similar for moderate- and vigorous-intensity activities. Comparing highest and lowest categories of physical activity, ORs for CP/CPPS were 0.77 (95\% CI $0.59-1.02$, p for trend 0.02) for vigorous activity, and 0.69 (95\% CI $0.43-1.10$, p for trend 0.01 ) for moderate activity. We also investigated the joint association of moderate and vigorous physical activity. Compared to men who had high physical activity levels of either moderate- or vigorous-intensity ( $>8.8$ MET-h/wk), those who engaged in high levels of both moderate- and vigorous-intensity activity did not demonstrate additional reduction in risk of CP/CPPS (P for interaction $=0.03$ )" [20].

Dikov D, et al: "Quantitative analysis of the number, normal and pathologic ratios between lymphocytes and epithelial cells (ECs), and the significance of intraepithelial lymphocytes (IELs) in normal prostatic epithelium, benign prostatic hyperplasia (BPH), and high grade prostatic intraepithelial neoplasia (PIN) in relation to NIH category IV prostatitis (histologic prostatitis: HP) was studied in autopsy prostate.

IELs were analyzed in 59 autopsy prostates, which was routinely embedded in paraffin and immune-histochemically stained for CD3. An average of 300-500 ECs were counted per case. The number of IELs was calculated as the mean $/ 100$ ECs. Category IV prostatitis was evaluated using NIH consensus grading system in terms of anatomical localization and grade.

In healthy individuals the mean number of IELs/100 ECs was $0.61 \pm 0.34 \%$ or $\leq 1$ lymphocyte $/ 100 \mathrm{ECs}$, which is considered as the normal basal level of prostate IELs. In category IV prostatitis, the mean number of IELs/100 ECs was $8.53 \pm 3.25 \%$ or $5-11$ lymphocytes/100 ECs. The number of IELs in both around and inside inflammation areas correlated to the grade and location of HP $(P<0.0001$ and $P<0.0003)$, the presence of acute glandular inflammation $(P<0.0001)$, the scattered stromal lymphocytes $(P=0.029)$, and BPH and PIN associated prostatic inflammation $(P<0.0001)$.

The study presents the first attempt to examine and score the basic quantitative values of prostatic IELs in normal prostate and in relation to category IV prostatitis. The detected normal upper limit of CD3+ IELs is 1 lymphocyte/100 ECs in the normal prostate epithelium. This is considered as an organ specific characteristic of the prostate-associated lymphoid tissue (PALT). Values >5 IELs/100 ECs indicate the presence of category IV prostatitis. The severity of 
inflammation correlates to the number of IELs. There is an intimate link between the quantity of the IELs, the degree of the severity and the localization of category IV prostatitis. HP is a chronic and dynamic inflammatory process affecting the whole prostate gland. The increased number of IELs suggests the immune or autoimmune character of category IV prostatitis, BPH and inflammatory preneoplastic (PIN) lesions in the prostatic tumor environment" [21].

Anim JT, et al: "Inflammation is a common finding in benign prostatic hyperplasia (BPH) and may be classified as acute, chronic active or chronic inactive prostatitis. The aim of the present study was to localize the different types of inflammatory cells in prostatic lesions to determine the sequence of events in the cellular reaction. We have carried out immunohistological characterization of the inflammatory cells, using CD45RO and CD3 antibodies to detect T-lymphocytes, CD20 antibodies to detect B-lymphocytes, CD68 to detect macrophages, kappa and lambda immunoglobulin light chains, and antibodies against prostate specific antigen (PSA) and prostate specific acid phosphatase (PSAP). Macrophages accumulated in the lumen and glandular epithelial layers of damaged prostatic glands and were found in the peri-glandular cuff of inflammatory cells in acute and chronic active prostatitis. Lymphocytes also accumulated in large numbers in the glandular epithelial layers and around the glands, indicating an association with macrophages. B-lymphocytes were scanty, if at all present, in acute and chronic active prostatitis, but were prominent within well-organized follicle centres in chronic active prostatitis. Cells positive for light chains were few and scattered in prostatic tissue. PSA and PSAP activity was lost in recently damaged prostatic glandular epithelium and reappeared only in regenerating secretory epithelium, indicating leakage as a result of damage. We suggest that the initial response to prostatic injury is cellular, and probably related to leakage into the peri-glandular tissues of PSA, PSAP and other antigenic molecules normally present in prostatic secretion. Macrophages respond, followed by recruitment of T-lymphocytes which participate in the inflammatory response and accumulate around the damaged glands. B-cell activity appears to be a late event" [22].

Pavone CA, et al: "Objectives: In this study we evaluated the association between chronic prostatitis syndrome (CPS), varicocele and hemorrhoids as manifestations of a pelvic venous disease. Our retrospective study was based upon 2,554 patients treated in two general urology clinics over the past 10 years. We have assessed the incidence of CPS among urological patients. We found 483 patients with CPS, representing $18.9 \%$ of the total number of visits at the outpatient clinic. In this group the percentage of varicocele and hemorrhoids was 14.69 and $8.48 \%$, whereas in a control group these figures were 5.02 and $5.84 \%$, respectively $(\mathrm{p}<0.001$ and 0.1054). Such a difference is statistically significant and suggests a higher prevalence of varicocele in the CPS group, but this may be due to a methodological error of the retrospective study. Only a prospective study, which is of importance due to the frequency of the disease, can give a precise answer to this question" [23].

And according article: Endogenous Archeological Sciences: Anatomy, Physiology, Neuroscience, Biochemistry, Immunology, Pharmacology, Oncology, Genetics as Instrument for A New Field of Investigation? Modern Global Aspects for A New Discipline “Open Access J Addict \& Psychol. 2018:

"And Observing also the difference in bipedal and in quadrupedal primates; the monkeys show the same rate in urinary infections like actual humans? They need the same amount of antimicrobials/year due by UTI infectious disease? and why?"[24].

James A Levine: "Sitting too much kills. Epidemiological, physiological and molecular data suggest that sedentary lifestyle can explain, in part, how modernity is associated with obesity, more than 30 chronic diseases and conditions and high healthcare costs. Excessive sitting-sitting disease-is not innate to the human condition. People were designed to be bipedal and, before the industrial revolution, people moved substantially more throughout the day than they do presently. It is encouraging that solutions exist to reverse sitting disease. Work environments, schools, communities and cities can be re-imagined and reinvented as walking spaces, and people thereby offered more active, happier, healthier and more productive lives.

\section{Sitting sickness}

Sedentariness and low NEAT may be important factors in obesity, but it is less obvious as to why sedentariness is causally associated with diabetes and 33 other chronic diseases and conditions. To illustrate the impact of sitting, in one experiment, healthy volunteers were provided with three meals and encouraged to remain sedentary thereafter. The same participants, on another occasion, were provided with similar meals and asked after they had eaten to walk for 15 minutes at $1 \frac{1}{2} \mathrm{mph}(2.4 \mathrm{kph})$. Continuous glucose monitoring showed that the short walks halved postprandial glycaemic excursion, regardless of when the meal was consumed. In this instance, and in several other experiments, the data underscore how prolonged sitting increases insulin resistance and that breaking up sitting time can improve glucose handling. These data provide a physiological rationale as to why sitting is associated with type 2 diabetes and gestational diabetes.

Thirty-five chronic diseases and conditions are associated with sedentariness, including frailty in the elderly, weight regain after therapeutic weight loss, hypertension, osteoporosis, malignancies such as breast and prostate cancer, cardiovascular disease, male erectile dysfunction, depression, and back and musculoskeletal pain. Improved physical activity 
also helps with addictions to alcohol, opiates and cigarettes. Chronic diseases and conditions associated with sedentary behavior impact approximately $70 \%$ of patients and the majority of US healthcare costs, and so the fiscal consequences of a sedentary lifestyle are enormous" [25].

Hadi Daneshmandi, et al: "Excessive sitting behavior is a risk factor for many adverse health outcomes. This study aimed to survey the prevalence of sitting behavior and its adverse effects among Iranian office workers.

This cross-sectional study included 447 Iranian office workers. A two-part questionnaire was used as the data collection tool. The first part surveyed the demographic characteristics and general health of the respondents, while the second part contained the Nordic Musculoskeletal Questionnaire (NMQ) to assess symptoms. Statistical analyses were performed using the Statistical Package for the Social Sciences software using Mann-Whitney U and Chisquare tests and multiple logistic regression analysis. The respondents spent an average of 6.29 hours of an 8-hour working shift in a sitting position. The results showed that $48.8 \%$ of the participants did not feel comfortable with their workstations and $73.6 \%$ felt exhausted during the workday. Additionally, $6.3 \%$ suffered from hypertension, and $11.2 \%$ of them reported hyperlipidemia. The results of the NMQ showed that neck (53.5\%), lower back (53.2\%) and shoulder (51.6\%) symptoms were the most prevalent problem among office workers. Based upon a multiple logistic regression, only sex had a significant association with prolonged sitting behavior (odds ratio $=3.084$ ). Our results indicated that long sitting times were associated with exhaustion during the working day, decreased job satisfaction, hypertension, and musculoskeletal disorder symptoms in the shoulders, lower back, thighs, and knees of office workers. Sitting behavior had adverse effects on office workers. Active workstations are recommended to improve working conditions" [26].

Loran $\mathrm{OB}$, et al: "33 patients with benign prostatic hyperplasia (BPH) were exposed to hyperbaric oxygenation (HBO) and SHF waves. Group 1 patients had no prostatic inflammation, group 2 patients had combination of BPH with chronic prostatitis. Both these modalities are considered as to mechanism of action at different periods of the development of urination disturbances. The combined therapy imposed a positive trend in urination parameters, especially evident in group 2 patients. In them mean urination frequency reduced from 14.8-8.5 times to 6.3, mean urine volume increased from 83.6 to $199 \mathrm{ml}$. Index I-PSS fell in group 1 by 2 and group 2 by 6.4 scores. L index of quality of life declined by 1.2 and 1.6 scores in groups 1 and 2, respectively. It is inferred that combined use of HBO and SHF therapy is highly effective in urination disorders in patients with BPH suffering from or without chronic prostatitis" [27].

Taoka R, et al: "We report that asymptomatic histological inflammation causes repeated destruction, healing, and regeneration of the prostate tissue, leading to the enlargement of prostatic nodules, while at the same time causing significant morphological changes to stromal tissue (remodeling), which can increase urination resistance and result in the condition changing to symptomatic BPH. Reactive oxygen species produced as a result of the low-oxygen environment of the remodeled tissue reportedly induce inflammatory and proliferative cytokine expression, which can promote a vicious cycle of remodeling.

The potential role of anti-inflammatory agents for symptomatic BPH drugs currently investigated for the treatment of prostatic inflammation include the hexaniclipidosterolic extract of Serenoa repens, nonsteroidal anti-inflammatory drugs, and so on. In particular, recent reports have stated that the anti-inflammatory effect of phosphodiesterase type 5 inhibitors (PDE 5i) is useful for the treatment of BPH. It is demonstrated that PDE5i leads to an increased testosterone/estradiol ratio. In an experimental study in the rabbit, PDE5i reduces prostate inflammation, fibrosis, and hypo-oxygenation. The clinical guidelines published by the American Urological Association and those of the European Association of Urology do not mention anti-inflammatory agents. Although further evidence is needed to support this, the underlying mechanism is currently being elucidated, indicating that it is a promising agent" [28].

Motoaki Saito, et al: "In the light of increasing evidence that benign prostatic hyperplasia is associated with cardiovascular disease, we have investigated the relationship between prostatic blood flow and prostatic hyperplasia in the spontaneously-hypertensive-rat (SHR). Twelve-weekold male SHRs were treated with nicorandil for six weeks. Wistar-Kyoto rats were used as controls. Six weeks after nicorandil treatment, blood pressure and the prostatic blood flow were estimated, and tissue levels of malondialdehyde, HIF- $1 \alpha$, TGF- $\beta 1$, bFGF, dihydrotestosterone, and $\alpha$-SMA were measured. SHRs showed significant increases in blood pressure, tissue levels of malondialdehyde, HIF- $1 \alpha$, TGF- $\beta 1$, bFGF, $\alpha$-SMA and a significant decrease in the prostatic blood flow. Although treatment with nicorandil failed to alter the blood-pressure and $\alpha$-SMA, it significantly ameliorated the increased levels of malondialdehyde, HIF- $1 \alpha$, TGF- $\beta 1$, and $\mathrm{bFGF}$. There were no significant differences in tissue levels of dihydrotestosterone among any groups. These data indicate that development of prostatic hyperplasia may be associated with prostatic hypoxia, which nicorandil prevents via its effect to increase the blood flow. The present study demonstrated that SHRs showed significant increases in blood pressure, tissue levels of MDA, HIF- $1 \alpha$, TGF- $\beta 1$, bFGF and $\alpha$-SMA, and a significant decrease in the prostatic blood flow. Although treatment with nicorandil failed to decrease the blood pressure, it ameliorated these factors and inhibited the development of ventral prostatic hyperplasia. We propose that development of prostatic hyperplasia is related 
to prostatic hypoxia, which nicorandil prevents via its ability to increase the blood flow in the prostate" [29].

Jason Gandhi, etal: "Dystrophiccalcifications of theprostate occur in accordance with a disturbance in phosphorous or calcium metabolism. These distinct calcifications manifest as tiny stones that can grow large enough to cause muscle cramps and pain in the groin. When the cell membrane leaks calcium ions, an acidic environment is created, and crystallization begins to occur. These conditions can be generally circumvented using inhibitors such as osteopontin, which prevent calcium deposition under natural conditions. The mechanism of action underlying HBOT for urothelium dystrophic calcification is not yet fully understood. It is presumed that HBOT facilitates the delivery of oxygen to tissues unable to heal in a calcium-ion driven acidic environment. In a study by Kern \& Humphreys, 2 a 71-yearold man with a history of dystrophic calcifications of the prostatic fossa, recurrent prostatitis, and urinary retention secondary to benign prostatic hyperplasia completed two rounds of HBOT following holmium-laser lithotripsy of the calcifications ( 37 sessions at 2.0 to $2.4 \mathrm{~atm}$ for the first round and 20 sessions at $2.0 \mathrm{~atm}$ for the second round). Results of this case demonstrate promise; following minor recurrent calcifications after the first round of therapy, the patient exhibited well healed prostatic fossa, a healthy urothelium, and marginal calcifications" [30].

Declan J McKenna, et al: "Hypoxia is a well-established characteristic of prostate tumors and is now recognized as a major contributory factor to both tumor progression and increased resistance to therapy. One strategy to target hypoxic tumor cells is the development of hypoxia-activated prodrugs (HAPs), which are activated in low oxygen environments. Several HAPs have been developed but despite encouraging results from preclinical studies many of these have performed disappointingly in clinical trials. In the developing era of precision medicine, it is clear that more strategic deployment of these agents is required, based on reliable methods that can identify patients who will benefit from HAP treatment, either alone or in combination with other drugs. This review discusses the primary limitations of using HAPs to treat hypoxic tumors and explains how these challenges can be addressed. In particular, it emphasises the importance of tumor imaging and identification of reliable biomarkers for measuring hypoxia and monitoring cellular response to treatment in individual patients. Developing predictive assays for clinical use will be paramount in demonstrating the patient impact and effectiveness of HAPs for personalized medicine.

A major challenge in cancer therapy is to develop therapeutic agents that selectively target tumor cells. One avenue towards the development of more selective cancer therapies is to exploit the unique physiological properties of solid tumors using prodrug approaches. Hypoxia generated as a result of a poor and inefficient neovasculature is a characteristic feature of many solid tumors and is associated with the development of an aggressive phenotype and resistance to radiotherapy and chemotherapy. Whilst problematical for conventional therapies, hypoxia is regarded as a valid target for drug development and a series HAPs have been developed over a period of 30-40 years with eight HAPs reaching clinical evaluation. Currently, no HAP has reached the market and this is somewhat perplexing given the overwhelming evidence of solid tumors containing significant levels of acute and chronic hypoxia. If patients were molecularly stratified for treatment based on their tumor hypoxia signature including analysis of reductase expression, it is possible that the HAPs in combination with chemotherapy or radiotherapy would have resulted in improved treatment outcomes. Prostate tumors are considerably hypoxic as discussed in this review, which poses some unique challenges to effective treatment of aggressive forms of this disease with standard therapies such as docetaxel and/or radiotherapy. Clinical trials carried out with AQ4N have been promising, demonstrating safe administration of a uHAP that rapidly distributes throughout the body and penetrates into hypoxic regions where it is bio-reduced to a persistent DNA-affinic topo II-targeting metabolite. The deuterated AQ4N analogue OCT1002 offers great potential in the treatment of prostate cancer, for example in the combination with ADT. In prostate cancer, uHAPs could also be used in combination with PARP1 inhibitors in patients whose tumors harbor DDR deficiencies. Much progress is being made on how best to utilize PARP1 inhibitors but prior analysis of tumor heterogeneity and target expression is vital for clinical success. For example, a recent phase 2 trial that concerned patients with metastatic prostate cancer benefitted from whole-exome sequencing and transcriptome analysis on DNA from fresh-frozen tumor-biopsy samples prior to treatment. In this study, understanding of DNA defects enabled clinicians to select patients suitable for the PARP inhibitor olaparib to ensure better treatment outcome. The emergence of genetic and hypoxic signatures and the ability to image and analyse the heterogeneity of prostate tumors provides new opportunities for employing HAPs and uHAPs in combination with molecularly-targeted agents and/or radiotherapy" [31].

Ficarra V: "In this issue of BJU International, Gandaglia, et al. summarize the evidence supporting the role of chronic prostatic inflammation in the pathogenesis and progression of BPH.

Briefly, one or more concomitant factors (bacterial infections, viruses, sexually transmitted organisms, dietary factors, hormones, autoimmune response and urine reflux) can stimulate an inflammatory reaction in prostatic tissue characterized by infiltration of T-lymphocytes, activation and up-regulation of pro-inflammatory cytokines, increased expression of potent stromal and epithelial growth factors (e.g. fibroblast growth factor, FGF-2) and consequently 
abnormal proliferation of prostatic cells. Local hypoxia plays an important role stimulating reactive oxygen species (ROS) release, Continuous repair gives tissue remodeling.

Neo-vascularization processes and the production of other additional growth factors (vascular endothelial growth factor, interleukin 8, FGF-7, TGF- b and FGF-2). Interestingly, this mechanism is self-perpetuating, creating a local vicious cycle.

Available clinical data seems to emphasise the prevalence of chronic prostatic inflammation in $\mathrm{BPH}$. Indeed, a subanalysis of the REDUCE (Reduction by Dutasteride of prostate Cancer Events) trial shows that in patients with $\mathrm{BPH}$ a chronic prostatic inflammation can be detected in $77 \%$ of patients who underwent prostate biopsies. This study also showed a statistically and clinically significant correlation between chronic prostatic inflammation and LUTS severity, especially when the storage subscale was considered. As extensively described by Gandaglia, et al., other studies have shown a significant correlation between chronic prostatic inflammation and prostate volume and an increase $\mathrm{d}$ risk of acute urinary retention. Chronic prostatic inflammation can be histologically detected only in patients who undergo prostate biopsies for suspicion of prostate cancer. Most patients with LUTS/BPH do not undergo a prostate biopsy. For this last category, the use of specific biomarkers correlated with chronic prostatic inflammation has been proposed as a potential alternative. Although interleukin -8seems to be the most reliable and predictive surrogate marker to identify patients with chronic prostatic inflammation, its use is not yet popular, it is expansive and probably requires further clinical evaluation before introduction into daily clinical practice. In this context, the detection of prostatic calcification scan represent a simple ultrasound sign to suspect the presence of chronic prostatic inflammation.

In patients aged $>50$ years, prostatic calcifications represent an age-related alteration of the prostatic fluid. Prostatic calcifications can produce an obstruction of the intra-prostatic ducts stimulating an inflammatory response in prostatic tissue characterized by lymphocyte infiltration, cytokine activation and ROS release. This results in damage of epithelial and stromal prostatic cells and a subsequent process of wound healing consisting of stromal proliferation and excessive extracellular matrix production; summarizes these mechanisms following prostatic duct obstruction. I think that in patients with prostatic calcifications and severe LUTS (with predominant storage symptoms) the presence of chronic prostatic inflammation should be strongly considered" [32].

Andrzej Górski, et al: "Our data suggest that phage therapy could be efficient in patients with prostatitis. Our study comprised 27 patients most of whom received phages intrarectally for an average of 47 days. Eradication of pathogen as confirmed by two consecutive EPS cultures was observed in 13 patients. A significant decrease in the EPS leukocyte count, significant reduction of the prostate volume and an increase in the maximum urinary flow were also noted. No significant side effects were observed. Additional studies have also reported encouraging findings. Optimal results have been achieved using intra-rectal phage administration. No reliable proof of phage penetration into human prostate is available; in rats phage may penetrate prostate following intravenous-administration [33].

Bartoletti R, et al: "We aim to evaluate the role of biofilmproducing bacteria in the clinical response to antibiotic therapy among patients affected by chronic bacterial prostatitis (CBP).

All patients attending our centre from January to December 2008 due to prostatitis-like symptoms with a positive MearesStamey test were enrolled. The clinical symptoms were assessed according to the NIH-CPSI, and the bacterial strains isolated from the patients enrolled were identified and tested for antibiotic sensitivity using cards of the Vitek II semiautomated System for Microbiology. Quantitative bacterial slime production was assessed by the Christensen microwellassay. All patients were treated with fluoro-quinolones for 4 weeks and reevaluated clinically and microbiologically after 3 months.

One hundred and sixteen patients were enrolled, and 150 bacterial strains were isolated from all patients. About $85 \%$ of these strains were strong or moderate biofilm producers. Patients with strong or moderate biofilm-producing bacteria had a higher NIH-CPSI symptom score than those without biofilm-producing bacteria (mean $17.6 \pm 5.6$ vs. $14.1 \pm 3.3$; $p=0.0009)$. At the follow-up, 68 patients $(58.6 \%)$ had negative microbiological tests, but only 11 (9.48\%) reported a reduction in NIH-CPSI score. Improvement of symptoms was found statistically significantly less frequent in patients with biofilm-producing bacteria than in those without ( $p$ $=0.03$ ). Ultra structural analysis showed cellular forms in active replication with aberrant morphology of unknown cause and confirmed strong slime production with consistent bacterial strati.

In our CBP population, biofilm-producing bacteria were commonly found and had a significant negative impact on the clinical response to antibiotic- therapy" [34].

Corvec S, et al: "Limited antimicrobial agents are available for the treatment of implant-associated infections caused by fluoroquinolone-resistant Gram-negative bacilli. We compared the activities of fosfomycin, tigecycline, colistin, and gentamicin (alone and in combination) against a CTXM15-producing strain of Escherichia coli (Bj HDE-1) in vitro and in a foreign-body infection model. The MIC and the minimal bactericidal concentration in logarithmic phase (MBC(log)) and stationary phase (MBC(stat)) were 0.12 , 
0.12 , and $8 \mu \mathrm{g} / \mathrm{ml}$ for fosfomycin, $0.25,32$, and $32 \mu \mathrm{g} / \mathrm{ml}$ for tigecycline, $0.25,0.5$, and $2 \mu \mathrm{g} / \mathrm{ml}$ for colistin, and 2,8 , and $16 \mu \mathrm{g} / \mathrm{ml}$ for gentamicin, respectively. In time-kill studies, colistin showed concentration-dependent activity, but regrowth occurred after $24 \mathrm{~h}$. Fosfomycin demonstrated rapid bactericidal activity at the MIC, and no regrowth occurred. Synergistic activity between fosfomycin and colistin in vitro was observed, with no detectable bacterial counts after $6 \mathrm{~h}$. In animal studies, fosfomycin reduced planktonic counts by $4 \log (10) \mathrm{CFU} / \mathrm{ml}$, whereas in combination with colistin, tigecycline, or gentamicin, it reduced counts by $>6 \log (10) \mathrm{CFU} / \mathrm{ml}$. Fosfomycin was the only single agent which was able to eradicate E. coli biofilms (cure rate, $17 \%$ of implanted, infected cages). In combination, colistin plus tigecycline $(50 \%)$ and fosfomycin plus gentamicin (42\%) cured significantly more infected cages than colistin plus gentamicin $(33 \%)$ or fosfomycin plus tigecycline $(25 \%)(P$ $<0.05$ ). The combination of fosfomycin plus colistin showed the highest cure rate (67\%), which was significantly better than that of fosfomycin alone $(P<0.05)$. the combination of fosfomycin plus colistin is a promising treatment option for implant-associated infections caused by fluoro-quinoloneresistant Gram- bacilli" [35].

David Lebeaux, et al: "A more recent example of an antibiotic associated with a potent antibiofilm effect is that of daptomycin Biofilm recalcitrance toward antibiotics is responsible for most of the difficulties encountered in the treatment of biofilm-related infections. Major advances have been made in the characterization of factors associated with this problematic biofilm property. Recognition of the role played by persister cells and the recent identification of several molecular mechanisms involved in the generation of per sisters have already led to several potential anti biofilm treatments. Validation of these new approaches will likely require renewed interactions between fundamental research and clinical practice before these approaches can be included in future therapeutic arsenals for use against difficult-totreat infections" [36].

Muhamad Abu Bakar, et al: Treatments aimed at disrupting biofilms": Microbial biofilm formation is responsible for the development of acute-to-chronic infection in several diseases including cystic fibrosis, periodontitis, infective endocarditis, persistent otitis media, chronic rhino sinusitis, chronic tonsillitis, prostatitis, chronic osteomyelitis, atopic dermatitis, onycho mycosis, dental caries, infectious kidney stones, and chronic wounds. Biofilms can also form on any surface, living or nonliving, even on clinical devices like pacemakers, implants, and catheters, and are very difficult to eradicate, which accentuates clinical consequences; for example, pseudomonal infections can affect any part of the human body. The microorganisms' adaptive capability and genetic changes within the biofilm lead to resistance to all known antimicrobial medicines.
Pseudomonal infections in particular become really difficult to be treated and can threaten human life. It is thought that $99 \%$ of the biosphere's bacteria live in and that microbial communities gain an advantage living in this state. Consequently, microbial biofilms are thought to significantly affect human health by increasing morbidity, mortality, and health care cost. Biofilms not only add to hospital-acquired infections (HAIs) by increasing their chronicity and persistence but also colonize in other areas of the environment instigating corrosion, fouling of water pipes, and food and pharmaceutical decomposition. Another study reported that micro bialbio films can stick onto and infect all medical devices such as orthopedic prostheses and intravascular catheters and promote up to $60 \%$ of HAIs.89Microorganisms in biofilms are distinctively more resistant to antimicrobial agents and environmental insults and are very difficult to eradicate. Biofilms in general (and chronic tonsillitis specifically) can lead to substantial economic costs for countries and individuals and health concerns and are an evolving public health problem in both high- and low-resource settings. The explosion of antibiotic resistance throughout the world of many microbial strains has put pressure on the research and medical communities to find an alternative strategy for the management of biofilmmediated diseases.

"Perhaps new antibiotics are not the only way to combat biofilm infections if we could make ineffective older antibiotic active again." In one study, a 2-amino-imidazole molecule was developed which was capable of disrupting biofilms through making microorganisms which were previously antibiotic-resistant more vulnerable to older antimicrobials.

Immunotherapy(usingcyclicdinucleotides) hasbeen effective in the management of different cancers, and this molecule has also been utilized as a therapeutic strategy for biofilm-related infections. Immunoprophylaxis and immunotherapy might provide new tools to combat Staphylococcus epidermis biofilm formation.

Multiple studies revealed thata 3,5-cyclic diguanylic acid(cdi-GMP)-binding protein was found in biofilm communities. BdcA (a protein that enhances biofilm dispersal) confiscates c-di-GMP and minimizes its local concentration and is partly responsible for the reduction and down regulation of EPSs of biofilms and for the up regulation of swimming, swarming, and planktonic microbes. This phenomenon has been observed in Pseudomonas sp. and Rhizobium melilotibiofilm communities. Multiple groups of scientists recently reported that CdrA (an adhesin compound) which is produced by biofilms in response to high levels of c-di-GMP binds with Psl and stabilizes biofilm structure. Multiple research studies have identified at least three extracellular polysaccharides (Alginate, Pel, and Psl) that are important factors for structure maintenance and antibiotic resistance of biofilm. Another study revealed that exogenous addition of d-amino 
acids disrupted preformed biofilms by disturbing adhesive fiber interactions and was also effective in preventing biofilm formation by Staphylococcus aureus and Pseudomonas aeruginosa.

Another research study reported that biofilmdisassembly molecule is norspermidine which has a similar dispersal mechanism to d-amino acids by targeting the exo polysaccharides. The biofilm-inhibiting properties of norspermidine were detected in S. aureus and Escherichia coli pellicle biofilm. Current research needs to focus on the development of norspermidine, BdcA, d-amino acids, and other polyamines as a novel antibiofilm approach, and medical communities should no longer depend exclusively on antimicrobials (which are increasingly ineffective with many pathogenic microorganisms because of resistance) and surgery to treat infectious diseases. Other studies have identified additional ways of disrupting biofilms. Bioactive enzymes such as dispersin or Proteinase $\mathrm{K}$ studied in orthopedic implants made bacteria more susceptible to antibiotics and finally eradicated the biofilm by affecting polymers or proteins of the biofilm structure.

Several cytotoxic agents have also been found to successfully eliminate biofilms from implant surfaces, with citric acid being reported to be the most successful in eradicating biofilms on titanium surfaces. Multiple research studies have identified that electrical current can successfully detach $S$. aureus and S. epidermis biofilms from stainless steel implants. Another study observed that biofilms of S. epidermis on stainless steel fasteners were successfully eradicated through pulsed electromagnetic fields in combination with gentamycin.

A new cluster of research studies have used lasergenerated shockwaves to effectively break up biofilms. The technique was performed using a Q-switched, ND: YAG rhythmically laser functioning at a "rep rate of $10 \mathrm{~Hz}$ with $1500 \mathrm{~mJ}$ pulses centered at $1064 \mathrm{~nm}$. The laser pulses were used to create shockwave pulses in $\mathrm{Al}$ coated polycarbonate substrates and a resulting peak stress of greater than 50 $\mathrm{MPa}$ " was able to reduce 55\% living microorganisms.

The laser technique offers another way of disrupting biofilms and is useful in the management of infected wounds, where standard treatment modalities such as topical antimicrobials or the removal of dead, damaged, or infected tissue are unsuccessful or injurious.

One study found that just $4-10$ seconds of the laser therapy was able to disperse $97.9 \%$ of $P$. aeruginosa from biofilms on nitinol stents to single-celled planktonic microorganisms that can be more easily treated with antibiotics. Another found that laser-generated shockwaves therapy quickly disrupts the biofilms in infected wounds to eliminate the microorganisms and intensify the effectiveness of topical antimicrobials in the residual biofilm. Such interventions will promote patients' quality of life by reducing healing times and morbidity, and save health care costs. $\mathrm{N}$-acetylcysteine (NAC) is an antioxidant mediator which reduces the variety of microbial bacteria on biofilm emergence and evolution, inhibits the manufacturing of the extracellular polysaccharide matrix, and promotes the disruption of mature biofilms. NAC has been found to reduce Streptococcus pneumoniae and Haemophilus influenza adhesion to human oropharyngeal epithelial cells in laboratory experiments. Chronic infections raise prostaglandin levels, and NAC effectively reduces these levels and helps to disrupt the biofilms. Correspondingly, aspirin-like non-steroidal antiinflammatory drugs (NSAIDs) decrease biofilm production and completely block fungal infections. NAC interacts with the sulfhydryl group of enzymes involved in EPS production or excretion, which reduces the activity of these molecules or inhibits cysteine utilization. NAC, decreases in vitro biofilm formation, and other research on salicylates shows a similar negative effect on the production of biofilm. A study which applied both found that therapeutic doses of acetylsalicylic acid (ASA) and NAC diminish tonsillar mucosal biofilm formation in chronic or recurrent tonsillitis. Another Iraqi study found a strong correlation between the biofilm of Streptococcus pyogenes and recurrent tonsillitis and that three types of vinegar eradicated streptococcal biofilm remarkably: date (100\%), apple (95.5\%), and grape (90.9\%). A later study also demonstrated the potential of vinegar in eradicating tonsillar biofilm. In a laboratory experiment, while washing and cleaning with a soft brush did not remove the chronictonsillitis biofilm layer on the tonsil surface, using a harder brush removed more biofilm. Researchers believe that the physical removal of biofilm (by brushing or using ultrasound activated bubbles) from the tonsil surface in vivo will lead to greater effectiveness of topical antimicrobials and decrease the need for systemic antimicrobials" [37].

Giovanna Batoni, et al: "Overall, biofilm-associated infections may be subdivided into two main categories. The first one involves biofilm formation on host tissues (e.g. epithelia, mucosal surfaces, teeth). Examples of this type of infections are pulmonary infections in cystic fibrosis (CF) patients, foot ulcer infections in diabetic patients, chronic otitis media orrhino sinusitis, chronic prostatitis, recurrent urinary tract infections, and dental caries or periodontitis. The second type of infections may arise from microbial colonization of abiotic surfaces such as those of indwelling medical devices. A crucial feature of biomaterial-associated infections is that microbial cells may detach from biofilms and disseminate to the surrounding tissues or to the bloodstream, further exacerbating the clinical outcome of the infection with the increased use of medical devices in health care procedures, biofilm-associated infections have emerged as a major problem indifferent clinical disciplines. It is estimated that up to $80 \%$ of microbial infections in the human body involve biofilm formation, greatly contributing to morbidity and mortality, especially in hospital settings. 
Biofilm-associated infections are difficult to prevent, diagnose, and treat. A relevant biofilm property is the severely reduced susceptibility to antimicrobials, which is considered a multifactorial process. Beside the classical resistance mechanisms, due to the acquisition of mobile genetic elements, other biofilm-specific resistance mechanisms have been proposed. These include: reduced diffusion or sequestration of antimicrobials through the extracellular matrix, low growth rate of biofilm cells, presence of dormant cells virtually tolerant to all drugs ("persisters"). The treatment of biofilmassociated infections is so burdensome that often the only option is to remove the colonized medical device or to undergo surgical debridement of the biofilm-infected tissue. Antibiofilm drug-development: properties of an "ideal" antibiofilm agent Biofilms are microbial communities that display unique characteristics compared with their planktonic counterparts.

\section{Display rapid killing ability}

Biofilms are highly dynamic entities that develop according a well-defined step-by-step process which roughly involves an initial adhesion phase followed by a maturation phase and a dispersal phase. The adhesion phase involves a primary attachment of free-floating cells to a conditioned surface by weak and reversible long-range interactions (e.g. electrostatic and hydrophobic interactions, steric hindrance, vander Waals forces, hydrodynamic forces and others). Conditioning occurs when a foreign body is exposed to body fluids and its surface is modified by the adsorption of host molecules (e.g. albumin, lipids, extracellular matrix molecules, complement, fibronectin, inorganic salts).

Following this, loosely bound microorganisms stably attach to the substratum by short-range and more specific molecular interactions between bacterial surface structures (e.g. pili, fimbriae, fibrillae, capsule etc.) and host molecules (e.g. fibronectin) that function as receptors. This is influenced by a number of factors: flow conditions, local environment, bacterial properties as well as the surface properties of the biomaterial/host tissue. Maturation of biofilms is associated with the production of EPS that constitutes a large proportion of the biofilm biomass and plays a major role in the establishment of the biofilm phenotype. A final step or dispersal phase involves the detachment of clusters of cells or single cells and colonization of surrounding sites. Due to this temporal biofilm heterogeneity, an ideal antimicrobial agent against biofilms should be able to act in a fast way, to face a rapidly changing entity, and to target cells before they stably enter in the biofilm community and switch to the biofilm phenotype. Act in different microenvironments and target slow growing or even non-growing cells. Mature biofilms are also spatially highly heterogeneous as gradients of oxygen, nutrients, $\mathrm{pH}$, and waste material are established due to the reduced diffusion of gasses and molecules through the extra cellular matrix. The establishment of microenvironments that differ for physicochemical characteristics may impair the activity of several antibiotics. Aminoglycosides fail to act in anaerobiosis and at low-pH, conditions found in the deep biofilm layers.

The establishment of gradients implies that cells in the biofilm's peripheryare directly in contact with oxygen and nutrients while those sited in the deepest biofilm layers may experience anoxia, lack of nutrients and acidic conditions. This generates a spatial and metabolic heterogeneity of the bacterial population that may include rapidly as well as slowly growing cells. Environmental conditions within a biofilm may induce the occurrence of dormant, non-dividing cells at high rate, the so-called "persisters", that constitute a small fraction of essentially invulnerable cells believed to play a key role in biofilm recalcitrance to antibiotics. An "ideal" antibiofilm agent should be able to act in disparate environmental niches and to target sub-populations of cells with different growth rate, including persisters. Molecules acting on multiple targets or able to synergize with antimicrobials displaying different mechanisms of action, could be suitable to accomplish this goal. Combination of an antibiotic acting on metabolically active cells (i.e. ciprofloxacin) with one able to target nondividing cells of Pseudomonasaeruginosa biofilms (i.e. colistin), was described to ensure higher biofilm killing rates in vitro as compared to the antibiotics used alone. The same combination also demonstrated some clinical efficacy in the early eradication therapy of intermittent airway colonization by $P$. aeruginosa in CF patients Penetrate the extracellular matrix and/or interfere with its production.

Typically, biofilm communities are encased in a selfproduced matrix of EPS that have been defined "the house of biofilm cells". EPS represents a major biofilm component accounting for up to $90 \%$ of the total biofilm dry biomass. It contributes to maintain biofilm architecture providing a highly hydrated environment and favoring cell-to cell and cell-to-surface adhesion. The major EPS components are polysaccharides, proteins, lipids and extracellular DNA (eDNA) distributed in a non-homogeneous pattern.

The matrix plays a central role in the biofilm resistance mechanism to antibiotics. It constitutes essentially a diffusion barrier that delays or prevents the interaction of antimicrobial agents with microbial cells. Depending on the charge of EPS and the drug, the latter may be sequestered or repulsed with consequent decrease of the bioactive concentration. Sub-inhibitory concentrations of some antibiotics may even induce matrix synthesis, as in the case of beta-lactam induction of alginate synthesis in P. aeruginosa biofilms or vancomycin induction of slime synthesis in coagulase-negative staphylococci. Ideally, an antibiofilm agent should be able to penetrate the matrix and/or to inhibit/interfere with its accumulation. The heterogeneity of matrix composition across different species/strains and the environmentally modulated expression of matrix 
synthesis, may render this requirement particularly difficult to be satisfied. Interfere with bacterial cell communication machinery Cells in biofilm communicate and coordinate their behavior through the secretion of signal molecules known as auto-inducers.

When the concentration of such molecules reaches a critical level (Quorum, Q) it is sensed by the population members (Sensing, S) that coordinate their behavior in a cell-density dependent manner. Different types of acyl homoserine lactones function as signal molecules in grambacteria, while in gram + ones QS-mediated gene expression is mainly triggered by small peptides. Different biofilmrelated properties are under the control of QS signals. Signal molecules may promote biofilm formation or, favor biofilm dispersal. The possibility to interfere with QS signals is an extensively investigated research area for biofilm control. As QS often regulates also the expression of virulence traits, the employment of antagonists or quenchers of QS signals could allow accomplishing the double goal of inhibiting biofilm formation and down-regulating pathogen's features involved in the pathogenic process. as QS quenchers not necessarily ensure that infectious bacteria are eradicated, their therapeutic use as a single treatment seems less feasible than their utilization in combination with sterilizing agents (e.g. antibiotics). The study by Christensen, et al. demonstrated a synergistic antibacterial efficacy of early combination treatment with tobramycin and QS inhibitors against P.aeruginosa in an intra-peritoneal foreign-body infection mouse model.

\section{Modulate host response to biofilm}

Biofilms are not only recalcitrant to antibiotics, but also evade host immune-responses. In vitro studies demonstrated that antibodies or phagocytic cells at most enter the interstitial voids (water channels) that intercalate the microcolonies in a mature biofilm, but barely penetrate the deep biofilm layers. Phagocytic cells seem not only to be unable to physically engulf the biofilm structures but also to be impaired in their activities.

The clinical outcome of biofilm-associated infections is often exacerbated by the intense host pro-inflammatory response, to the persistent microbial stimulus, that may greatly contribute to the tissue damage. Targeting macrophages 'pro-inflammatory activity can overcome the local immune inhibitory environment created by the biofilm and represent a novel immunotherapeutic anti-biofilm strategy. The nature of the biomaterial (MD) is also important for the host response to the combined presence of bacteria and biomaterial. Murine or human macrophages exposed to various biomedical polymers including polydimethy-lsiloxane (PDMS), expanded polytetra fluoro-ethylene(ePTFE), and alginate react by secretingproinflammatory cytokines such as interleukin (IL)-1 $\beta$, IL6,tumor necrosis factor (TNF)- $\alpha$, and IL-12. Similarly to other persistent infections, a balance between pro-inflammatory and anti-inflammatory immune cell responses is needed for an optimal control of biofilm infections. Ability to recruit immune cells and/or modulate the host immune response would be an added value of an ideal anti-biofilm drug. Synergize with other conventional and unconventional antimicrobial compounds. It is quite unlikely that a single drug may exhibit all the above reported features at once. Combinatorial therapeutic strategies may represent a valid approach to target the numerous features of the biofilm mode of life.

\section{Examples include}

The use of antibiotics in combination with compounds able to i) digest or destabilize the biofilm matrix; ii) inhibit QS signals; iii) interfere with pathways leading to persistency; iv) promote ROS production to increase bacterial membrane permeability; $\mathrm{v}$ ) rise the $\mathrm{pH}$ to enhance activity of aminoglycosides; vi) render antibiotics more accessible into biofilms. Rational of using antimicrobial peptides as therapeutic strategies against microbial biofilms. In the last decade, growing interest has been devoted to the possible use of AMPs as anti-biofilm agents.

AMPs may have the potential to exert activity against biofilms as they display many, if not all, the properties of an "ideal" anti-biofilm drug outlined in the previous section. They overall exert a fast killing ability.

Our previous studies focused on the antibacterial properties of the human betadefensin-3 (hBD3) against multidrug-resistant nosocomial bacterial strains, demonstrated that the peptide is bactericidal against a wide variety of gram+ and gram- bacteria in 1 to $20 \mathrm{~min}$, depending on the species.

Similar short killing times were obtained by us also testing a number of frog-skin derived peptides against bacterial strains isolated from hospitalized patients. This feature is a direct consequence of AMPs' main mechanism of action, namely, per meabilization of bacterial membranes.

AMPs may have the potential to kill not only metabolically active cells but also slow growing or even persister cells. Cationic membrane-penetrating peptides containing various numbers of arginine (Arg) and tryptophan (Trp) repeats were demonstrated to be effective in killing planktonic persister cells of Escherichia coli HM22, a hyper-persister producer. Some of the Trp/Arg containing AMPs were also able to disperse and kill preformed biofilms harboring high percentages of persister cells.

Bacteria within biofilms resemble stationary phase bacteria and are generally less sensitive to antibiotics thanlog-phase bacteria. Two LL-37 analogues (P60.4Acand P10) significantly reduced the number of biofilm-derived cells at a concentration as low as $1.6 \mathrm{Mm}$ after $4 \mathrm{~h}$ incubation, suggesting the potential of such peptides to kill also bacteria released from the biofilm during the dispersal phase. 
Although most AMPs act, principally, by the electrostatic attraction to negatively charged bacterial surfaces followed by membrane disruption, their antibacterial activity may also involve interference with metabolic processes or with different types of intracellular targets that may result in inhibition of cell wall, nucleic acid or protein biosynthesis. The complex, often-multimodal, antimicrobial action of AMPs renders more difficult for microbes to develop durable resistance mechanisms, offering another notable advantage of AMPs over conventional antibiotics. This properly may allow AMPs to target different biofilm sub-populations and, in mixed biofilms, even different microbial species. AMP activity may be also modulated by environmental conditions.

We demonstrated that, similarly to other histidin-rich peptides, the antimicrobial properties of the human liverderived peptides hepcidin 25 and hepcidin 20 (hep-20) against clinically relevant bacterial and fungal strains are highly enhanced and quickened at acidic $\mathrm{pH}$. Besides this, we found that $\mathrm{pH}$ influences the mode of hepcidin 25 and hep-20 action on E. coli cells and model membranes, with a predominant membrane permeabilizing effect at acidic $\mathrm{pH}$ and a plausible effect on intracellular target(s) at neutral $\mathrm{pH}$. Acidic microenvironments may very well exist in the deep layers of a mature biofilm, due to the production of metabolites by bacteria, while at the biofilm periphery or in the close proximity of the water channels $\mathrm{pH}$ might be neutral. Acidic conditions may also originate in pathological conditions associated with biofilm formation such as those establishing in the dense mucus of the airway surface of $\mathrm{CF}$ patients.

Ability of AMPs to target biofilm-specific features has also been demonstrated. AMPs may act as QS inhibitors, downregulators of extracellular matrix biosynthesis or interferers of regulatory pathways that lead to the persister phenotype. AMPs can also act as immune-modulators, recruiting polymorphonuclear cells, lymphocytes or dendritic cells at the site of infection, enhancing the activities of host immune cells and modulating the release of pro or anti-inflammatory cytokines. Many AMPs interact with high affinity with the lipopolysaccharide (LPS) or endotoxin on the cell wall of gram- bacteria or in suspension and thus have the potential to neutralize the toxic effect of this molecule which is one the major mediators of septic shock. AMPs can promote healing processes by stimulating cellular proliferation or angiogenesis, thus potentially contributing to tissue repair during the course of biofilm infections. Other AMP-properties useful: the possibility: i) to immobilize the non-biomaterial surface to inhibit microbial adhesion; ii) to encapsulate them into natural or synthetic polymeric carriers as delivery systems. In the case of biomaterial-associated infections, the possibility of releasing the active molecule directly at the site of implant in a controlled manner may allow not only to prevent biofilm formation, but also offer the unique advantage to eradicate bacteria in peri-implant tissue; iii) to chemically manipulate them (amino acid substitution, introduction of D or non-natural amino acids, expression as fusion proteins, combination of different functional domains, others) in order to improve their effectiveness against planktonic and/or biofilm cells. Strategies combining AMPs with compounds acting with different mechanisms of action and targeting distinct biofilm features might represent a valid therapeutic approach to improve the AMP anti-biofilm potential. It is well established that AMPs may synergize with conventional and unconventional compounds, thus reducing the active antibiotic concentrations and possible side effects.

We recently focused on the study of the anti-biofilm properties of the frog-skin derived peptide temporin B (1Tb) in combination with conventional antibiotics or unconventional antimicrobial compounds such as cysteine or EDTA. A striking ability of the peptide to kill both forming and mature Staphylococcus epidermidis biofilms was observed, especially when used in combination with cysteine or EDTA. Temporin B in combination with EDTA was able to eradicate mature $\mathrm{S}$. epidermidis biofilms formed in vitro on silicon catheters suggesting the possible use of temporin BEDTA combinatorial strategies in the lock therapy of central access devices colonized by S. epidermidis biofilms. Some peptides can interfere with the early events of biofilm formation by preventing adhesion of bacterial cells to the substrate or to other cells, or by killing cells before they stably become part of the biofilm architecture. Others may act on established biofilms by killing mature biofilm cells, or by causing their detachment.

Interference with QS or other regulatory signals, dys regulation of genesinvolved in motility, modulation of theimmune system, interference with matrix synthesis/accumulation are other examples of AMP-anti-biofilm mechanisms of action. AMP-ability to inhibit biofilm formation or reduce/eradicate mature biofilms at concentrations equal or higher than its minimal inhibiting concentration (MIC) against the corresponding planktonic cells, may suggest that the peptide acts by a classical "microbicidal" effect.

In this second group of peptides, falls the human cathelicidin LL-37whose anti-biofilm properties have been extensively investigated. Over Hage, et al. assessed the mechanisms of P.aeruginosa biofilm inhibition by LL-37 by microarray technology and demonstrated that the peptide affects biofilm formation by decreasing the attachment of bacterial cells, stimulating twitching motility, and influencing two major QS systems (Las and Rhl), leading to the down regulation of genes essential for biofilm development.

Dyregulations of genes involved in biofilm formation and/or motility, only partially overlapping with those caused by LL-37, were also observed treating P.aaeruginosa biofilms with the small cationic peptide 1037 , suggesting that different peptides may target common set of genes controlling biofilm properties.HBD3 is another AMP possibly acting against biofilms by a "nonclassical" Mechanism-of action. 
Real-time polymerase chain reaction experiments demonstrated that the peptide interferes with the expression of ica A and ica D genes, which are part of the ica operon responsible of the synthesis of PIA (polysaccharideintercellular-adhesin), the major extracellular polysaccharide produced by a large fraction of staphylococcal strains. In the same study hBD3 was also demonstrated to up-regulate the expression of ica $\mathrm{R}$ (a transcriptional repressor of the ica operon expression) resulting in a marked attenuation of biofilm production. While evaluating the anti-biofilm properties of hep-20, a peptide with structural similarity with hBD3, our group evidenced that in the presence of hep-20 S. Epidermidis strains develop biofilms with an altered architecture and reduced amount of extracellular matrix. Due to its cationic nature, hep-20 could intercalate between the negatively charged bacterial cells interfering with the interactions of EPS components either reciprocally or with the cell-wall, thus reducing the amount of EPS that accumulates.

A striking ability of hepcidin to bind DNA has been recently reported. As eDNA is involved in biofilm development, it is tempting to speculate that binding of hep-20 to DNA may play a role in the destabilization of biofilm structure and accumulation of matrix components. Anti-biofilm action has been demonstrated for the synthetic cationic peptide IDR1018.

The peptide completely prevented biofilm formation and led to the eradication of mature biofilms of clinically relevant bacterial species, including P. aeruginosa, E. coli, A. baumannii, Klebsiella pneumoniae, methicillin resistant $S$. aureus, Salmonella typhimurium and Burkholderia cenocepacia. The mechanism of action involved the inhibition of a widely conserved stress response, the so-called stringent response, mediating (p) pp Gppsynthesis in response to environmental signals such as nutritional limitations. Coexistence of more activity, intensive structure-activity studies are still required for AMPs exploitation as anti-biofilm drugs. Interactions of antimicrobial peptides with biofilm component sand their role in biofilm resistance to AMPs Despite the numerous features underlined above render AMP promising antibiofilm agents, AMPs' clinical use is still hampered by several drawbacks that lower their translational potential. These may include potential toxicity at the therapeutically efficacious doses, poor stability in biological fluids, high production costs, potential development of resistance mechanisms, and/or unwanted interference with hostimmune responses. Interaction of AMPs with extracellular DNA, a common component of a variety of bacterial and fungal biofilms Extracellular DNA (eDNA) is a major component of the biofilm matrix of many pathogenic bacterial and fungal species. It mainly derives from cells undergoing lysis, but active mechanisms of secretion have been suggested as well. It may constitute a flexible pool of genes that bacteria in the biofilm exchange by mechanisms of horizontal gene transfer with possible acquisition of virulence traits and/ or resistance determinants. In P.aeruginosa biofilms the release of eDNA is under the control of QS signals and it may facilitate the twitching motility-mediated biofilm expansion by maintaining coherent cell alignments. A role of eDNA as nutrient source or cation chelator has also been proposed. Ability of sub-optimal antibiotic concentrations to promotee DNA release by biofilm cells has been reported. Kaplan et al. demonstrated that sub-inhibitory concentrations of beta-lactam antibiotics induce eDNA release and, in turn, cell aggregation and biofilm formation in some strains of $\mathrm{S}$. aureus. Similarly, biofilms of $S$. epidermidis pre-exposed to sub-inhibitory concentrations of vancomycin were found to contain higher concentrations of eDNA as compared to untreated biofilms and to impede penetration of the same antibiotic. Due to the crucial importance of eDNA in promoting cell aggregation and biofilm stabilization, several DNA-targeting anti-biofilm strategies have been proposed. Recombinant human DNase I was shown to prevent the formation and cause the detachment of staphylococcal biofilms at clinically achievable concentrations and to increase the survival of $S$. aureus-infected Caenorhabditis elegans nematodes treated with tobramycin compared with control nematodes treated with tobramycin alone. DNase I was reported to prevent biofilm formation of P.aeruginosa and to dissolve mature biofilms $(12,36$, and $60 \mathrm{~h}$ old) of the same species. Ciprofloxacin-loaded poly (lactic-co-glycolic acid) nanoparticles were functionalized with DNase I and their anti-biofilm activity was assessed against P.aeruginosa biofilms.

DNase Inactivated nanoparticles successfully reduced established biofilm mass, size, and living cell density. It is well established that by virtue of their cationic nature, several AMPs have DNA-binding properties. Mulcahy, et al. reported that in P.aeruginosa, DNA ability to bind and sequester cations, including magnesium, in the surrounding environment induces the PhoPQ and PmrAB two-component systems. Such systems regulate the cationic antimicrobial peptide (CAP) resistance operon PA3552-PA3559 whose induction leads to the expression of genes involved in LPS modifications (addition of amino-arabinose to lipid A), which, in turn, reduce the outer membrane permeability to CAPs. Biofilms supplemented with eDNA were 8-fold more resistant to CAP (polymyxin B, colistin) and 64-fold more resistant to aminoglycoside (gentamycin, tobramycin) than biofilms without exogenous DNA. The presence of DNA in the extracellular matrix of biofilms creates a localized cationlimited environment that is detected by P.aeruginosa leading to the induction of LPS modification genes and resistance to antimicrobials. Such a resistance mechanism may be widely used by bacteria to evade host innate immune responses or antimicrobial therapy in DNA-rich environments like biofilms or the lung of CF patients.

Interaction of AMPs with alginate and other polysaccharides, 
major components of biofilms of P.aeruginosa and other pulmonary pathogens exo polysaccharides are a major fraction of the biofilm matrix of a wide variety of microbial species. Most of them are long molecules, linear or branched, with molecular masses ranging from $0.5 \times 106$ to $2 \times 106 \mathrm{Da}$. They can be homopolysaccharides or, more often, hetero-polysaccharides that consist of a mixture of neutral and charged sugar residues. Most of the known exo polysaccharides are polyanionic (e.g. alginate of P.aeruginosa), but positively charged exo polysaccharides also exist (e.g. staphylococcal PIA). Polysaccharides play essential roles in the biofilm lifestyle.

Together with other EPS components, they allow the initial colonization of biotic and abiotic surfaces by planktonic cells, and the long-term attachment of mature biofilms to surfaces, maintain a highly hydrated microenvironment around biofilm organisms, form the skeleton and mediate the mechanical stability of biofilms, allow the accumulation of nutrients and the stabilization of extracellular enzymes. They confer resistance to non-specific and specific host defenses during infection and tolerance to various antimicrobial agents.

In the lung of CF patients P.aeruginosa undergoes complex adaptation process driven by genetic variation and acquires phenotypic diversity including the ability to synthetize high amounts of the extracellular polysaccharide alginate (mucoid strains). Alginate is a high-molecular-mass, linear polymer composed of D-mannuronic acid and L-guluronic acid. Polysaccharides other than alginate are produced by nonmucoid P.aeruginosa strains, the first to colonize the lung of CF patients. These polysaccharides include Pel, mainly composed of glucose, and Psl, a repeating pentasaccharide consisting of Dmannose, L-rhamnose, and D-glucose. Mucoid and non-mucoid P.aeruginosa strains differ by the qualitative composition of their polysaccharides in the biofilm matrix, predominantly alginate or $\mathrm{Psl} / \mathrm{Pel}$, respectively. Other pathogens produce considerable amount of extracellular polysaccharides. Members of the Burkholderia cepacia complex, or K. Pneumoniae, an important cause of nosocomial infections in infants or immune compromised patients.

Chan, et al: Who demonstrated that alginate induces a $\alpha$ helical conformation in a series of synthetic cationic AMPs, provided that the average core sequence hydrophobicity exceeds a "hydrophobicity threshold". Alginate both binds and promotes the self-association of the cationic peptides. Authors suggest that alginate might function as an "auxiliary membrane" for the bacteria encased in a biofilm. By forming complexes with peptides competitively with the bacterial membranes, alginate might entrap AMPs before they can reach their bacterial target and constitute for encased bacteria a protective environment against the innate host defense.

By Herasimenka, et al. studied the interaction of two cathelicidins, LL-37 and SMAP-29, with three bacterial polysaccharides, respectively produced by P.aeruginosa (alginate), B. cepacia (cepacian) and K. pneumoniae (capsular polysaccharide K40). The less inhibiting poly-saccharide was cepacian that even at high concentrations $(500 \mu \mathrm{g} / \mathrm{ml})$ caused an increment of the MIC value of not more than 2-4-fold as compared to that obtained in its absence. Alginate exerted a potent inhibitory effect on SMAP-29, LL-37, Bac7 and hBD3 causing a MIC increase of 8-32-fold, in the concentration range $100-500 \mu \mathrm{g} / \mathrm{ml}$. The inhibitory effect of $\mathrm{K} 40$ was also potent against PG-1, with a 8-32-fold MIC increment, while its effect was modest, although still significant, on the other peptides. Inhibition of peptides' killing ability by polysaccharides was overall fast and paralleled the polysaccharides' ability to inhibit E.coli inner membrane permeabilization. Intensive structure-function studies will constitute the basis for the design of optimized peptides with low tendency to interact with extracellular polysaccharides and/or high ability to penetrate them. While it has been reported that increasing the average core hydrophobicity of a cationic peptide can improve its antimicrobial activity, this same approach may promote peptide-peptide interaction in the weakly hydrophobic alginate, with consequent peptide aggregation and inactivation. A balance between antimicrobial activity and alginate permeability may represent a better strategy in future design of peptides active against P.aeruginosa biofilms. Of note, Yin, et al. recently demonstrated that although D-isomers of cationic interaction of AMPs with PIA of staphylococcal bio films S. aureus represents a major cause of health-care related and community-associated infections.

The worldwide emergence of multi-drug-resistant strains such as methicillin-resistant S. aureus (MRSA) has highly contributed to the spread of this bacterium. S. aureus often forms matrix-encased biofilms on tissues and medical devices, which confers an additional level of drug resistance and further complicates the treatment. S.epidermidis, considered an innocuous colonizer of the human skin, it is also frequently involved in nosocomial infections mostly occurring in patients with implanted medical devices such as intravascular catheters, prosthetic heart valves or orthopedic implants. Ability of S.epidermidis to colonize and form biofilms on a variety of biotic and abiotic surfaces is considered the major virulence factor of the bacterium. The phase of intercellular aggregation in staphylococciis mainly mediated by PIA, a polymer of $\beta$-1, 6 -linked N-acetylglucosamine with partially $\mathrm{N}$-deacetylated amine groups also called PNAG (poly-N-acetyl-glucosamine). De-acetylation of around $10-20 \%$ of the $\mathrm{N}$-acetyl-glucosamine residues produces free amino groups that become positively charged at neutral to acid $\mathrm{pH}$, resulting in a positive net charge of the otherwise neutral PIA molecule. The quite unusual net positive charge of PIA promotes intercellular interactions by binding to the negatively charged surface of bacterial cells. The biosynthesis of PIA is regulated by genetic elements located in the ica (intercellular adhesin) operon which comprises 
four genesica A, icaD, icaB and icaC. A gene, icaR, located upstream of ica $\mathrm{ADBC}$, encodes a transcriptional repressor of the ica ADBC-operon. ica gene expression and PIA production may also be subjected to environmental-driven regulation. It has been reported that anaerobic conditions induce PIA expression in both $S$. aureus and S. epidermidis. Mechanisms of phase variation regulating the on/off switching of the genes involved in PIA production have also been proposed. Isogenic mutants of wild-type biofilm-forming strains in which the ica locus has been mutated or deleted fail to form biofilms in vitro, demonstrating that the ica genes, and PIA production, are required for biofilm formation. Evidences support the view that staphylococcal PIA plays a crucial role in bacterial protection against major components of the human innate immune system, including host defense peptides. An icanegative S.epidermidis mutant strain, lacking PIA production, was demonstrated to be significantly more susceptible than wild typestrain to hBD3 and LL-37 suggesting that the positive net charge of PIA may cause electrostatic repulsion of positively charged peptides. PIA positive charge in resistance to cationic peptides is supported by the fact that inactivation of the icaB gene, whose product is responsible for PIA de-acetylation, reduces the positive charge of the polymer and increases the susceptibility of the icaB mutant strain to cationic peptides, at levels comparable to those obtained for the ica-negative mutant, devoid of PIA. Compared to wild-type S.epidermidis, the icaB mutant strain, with non-de-acetylated PIA, was highly impaired in its ability to form biofilms and to establish device-related infection in a murine model.

Despite the evidence that PIA, and possibly other staphylococcal polysaccharides, may play an inhibitory role on the antimicrobial activity of AMPs, ability of AMPs to inhibit PIA biosynthesis/accumulation has also been demonstrated. hBD3 was shown to downregulate the expression of icaA and icaD genes and upregulate that of icaR, while hep-20 was demonstrated to cause the formation of S.epidermidis biofilms with an altered architecture and a reduced amount of PIA at sub-inhibitory concentrations. S.epidermidis biofilms obtained in the presence of hep20 , were found to be more susceptible to vancomycin than control biofilms; the antibiotic, used at sub-lethal concentrations, caused a statistically significant reduction of biofilm-associated viable cells, as compared to biofilms not pre-treated with hep-20. Interference with extracellular matrix production/accumulation by hep-20, may improve diffusion of conventional antibiotics (e.g., vancomycin) through the biofilm layers.

Although the biosynthetic machinery for PIA production has been mainly investigated in staphylococci, homologous systems are present in other pathogenic, biofilmforming microorganisms, such as E. coli, Yersinia pestis, Aggregatibacter actinomycetemcomitans, and Bordetella pertussis. This suggests that the use of AMPs able to interfere with PIA biosynthesis/accumulation, alone or in combination with conventional antibiotics, could be a strategy to target biofilms of different medically important microorganisms. The biofilm matrix contains also a considerable amount of extracellular proteins.

Among these there are enzymes involved in the degradation of EPS (e.g. DNases, proteases, lipases, polysaccharideoroligosaccharide-degrading enzymes),with a role in nutrient acquisitio nor in the detachment and dispersal of biofilm cells, as well as non-enzymatic matrix proteins that exert structural functions, playing adhesive roles in the cell-to-surface or cellto-cell interactions. Proteins of the extracellular bacterial appendages such as flagella, type IV pili or fimbriae may also be part of the biofilm matrix and act as additional structural elements by interacting with other EPS components. For example, type IV pili of P.aeruginosa bind DNA and possibly work as inter-connecting structures. In staphylococci, although PIA is essential for biofilm formation of ica positive strains, significant matrix composition variation has been reported across clinical isolates and it is now clear that also ica-negative strains may exhibit marked biofilm-forming abilities.

Biofilm accumulation in these isolates is proteindependent as their biofilms are sensitive to protease treatment, but resistant to polysaccharide degrading enzymes: S.aureus surface protein C and G (SasC and SasG), the clumping factor $B$ (ClfB), the biofilm-associated protein (Bap),or the fibronectin/fibrinogen-binding proteins (FnBPA and FnBPB). In S.epidermidis, a accumulation-associated protein (Aap) contributes to both the primary attachment phase and the establishment of intercellular connections by forming fibrils on the cell surface. Staphylococcal peptides able to disrupt interaction of biofilmmatrix molecules with the bacterial surface have also been described. There is a family of short peptides called phenol-soluble modulins (PSMs), characterized by an amphipathic $\alpha$-helical structure and surfactant-like properties, the production of which is strictly regulated by the agr (accessory gene regulator) locus. PSMs includes the PSM $\beta 1$ and PSM $\beta 2$ that at low concentrations facilitate the formation of channels in the biofilm structure, thus promoting biofilm formation, while at high concentrations cause the detachment of biofilm cells independently of the nature (exo polysaccharidic or proteinaceous) of the biofilm. PSM $\beta 1$ and PSM $\beta 2$ were found to promote dissemination of biofilm cells from colonized catheters in a mouse model of device-related infection, while the use of antibodies against PSM $\beta$ inhibited bacterial spread from the device. Structural non-toxic analogs of the surfactantlike S.epidermidis $\beta$ subclass of PSMs, could be employed to promote dispersal of biofilm cells and favor their targeting by other bactericidal agents used in combination. Specific PSMinhibitors could aid in interfering with biofilm detachment phase and prevent dissemination of biofilm-associated infections. By using proteomic approaches, recently Gil, et 
al. characterized the exoproteome of exopolysaccharidebased and protein-based biofilm matrices produced by two clinical isolates of S. aureus. They found that, independently of the nature of the biofilm matrix, a common set of secreted proteins is contained in both types of exoproteomes. Notably, immunization with a bio filmmatrix exo proteome extract effectively reduced biofilm formation and the number of cells in the surrounding tissues in an in vivo model of meshassociated biofilm infection: potential of using extracellular proteins for anti-biofilm vaccine development.

A role of the Vibrio cholerae biofilm associated extracellular matrix protein Bap1 in cross-resistance to AMPs has been recently demonstrated by Duperthuy, et al. according to which growing a V. cholerae strain with sublethal concentrations of the cationic polypeptide polymyxin $B$ induces the release of outer membrane vesicles (OMVs) able to bind Bap1 at high levels through the OMV-associated major outer membrane protein OmpT. Bap1 then serves as an adapter protein between LL-37 and the OmpT on the surface of the OMVs. Following LL-37 binding by Bap1, the concentration of free LL-37 is reduced to sub-lethal concentrations, leading to the apparent resistance and survival of V. cholerae. Degradation of AMPs by biofilm matrix exoproteases might also bepossible. Our group has previously shown that proteases secreted by Porphyromonas gingivalis, an oral pathogen found in the sub-gingival biofilm of patients suffering from periodontitis, may degradehBD3 and inhibit the antibacterial activity of the peptide which is found at lower levels in the crevicular fluid of patients than in healthy controls. It should be mentioned here that often also host proteins might enter in the constitution of the bio film matrix. For instance, salivary protein sand glycoproteins are included in the extracellular matrix of oral bio films and are used as endogenous nutrients by plaque bacteria. The airway fluid of CF patients contain significant amount of filamentous (F)-actin that is released together with DNA from neutrophils and other cells that undergo lysis as the result of inflammation. Such host components may also interact with AMP sand cause loss of their antibacterial function. To date the diagnosis and treatment of biofilm infections in clinical settings is far from being satisfactory. IS necessary in the years to come to translate the huge amount of data obtained from extensive biofilm research into the clinic and to solve the numerous obstacles that still hamper the successful management of biofilm-associated infections.

AMP-EPS interactions could even be exploited for the design of AMP-based antibiofilm strategies aimed at sequestering essential EPS components, thus interfering with the establishment and maintenance of biofilm architecture. Specifically designed anti biofilm peptides could be employed to interfere with signaling pathways involved in the synthesis of EPS components. Extensive structure-function studies are desirable to identify the minimal structural features required for an optimal anti biofilm effect, since those known to enhance antimicrobial activity against planktonic cells not necessarily can be applied to biofilms. In other's and our experience, subtle changes in the amino acidic sequence of a peptide may greatly affect its antibiofilm activity.

A computational approaches implemented with large biofilm-oriented AMP-datasets, may help to predict novel peptide sequences specifically active against biofilms, while physicochemical inspired molecular modeling methods may provide insights on the AMPs antibiofilm mechanisms of action and/or interaction with EPS components.

The study of possible combination strategies is another research field that is worth investigating as the heterogeneity of microbial biofilms might require targeting cells in different metabolic state or environmental niches. Promising combinatorial strategies include AMPs with: i) other AMPs; ii) conventional drugs used for anti-infective therapy; iii) compounds that can dissolve the biofilm matrix(e.g. DNase, matrix-disrupting enzymes); iv) inhibitors of QS or other signal pathways; v) anti-inflammatory ormucolytic agents (e.g. ibuprofen, salicylic acid, $\mathrm{N}$-acetyl-cisteine). The advent of nanotechnology in the area of infectious diseases may offer further opportunities to optimize anti-biofilm AMP-activity. encapsulation of peptides or proteins in nano-carriers is emerging as a promising technology to overcome the poor stability of the active molecules in physiological medium, avoid their unwanted interactions with biofilm matrix components and deliver them directly to their microbial targets.

Our group has recently developed a delivery system based on the use of chitosan nanoparticles loaded with the antimicrobial peptide temporin B. We found that beyond the intrinsic antimicrobial activity of either chitosan nanoparticles or temporin B alone, the loaded nano carrier exhibited a highly enhanced and long-lasting microbicidal activity against a number of clinical isolates of S. epidermidis, while reducing the toxic potential of the encapsulated peptide against mammalian cells" [38].

Hogan S, et al: "Staphylococcal infections involving biofilms represent a significant challenge in the treatment of patients with device-related infections. Staphylococcus aureus biofilms have been shown to be SaeRS regulated and dependent on the coagulase-catalyzed conversion of fibrinogen into fibrin on surfaces coated with human plasma. Here we investigated the treatment of staphylococcal biofilm device-related infections by digesting the fibrin biofilm matrix with and without existing antimicrobials. The fibrinolytic agents plasmin, streptokinase, and nattokinase, and TrypLE, a recombinant trypsin-like protease, were used to digest and treat $\mathrm{S}$. aureus biofilms grown in vitro using in vivo-like static biofilm assays with and without antimicrobials. Cytotoxicity, the potential to induce a cytokine response in whole human blood, and the risk of induction of tolerance to fibrinolytic 
agents were investigated. A rat model of intravascular catheter infection was established to investigate the efficacy of selected fibrinolytic agents in vivo under biomimetic conditions, the fibrinolytic agents effectively dispersed established Saureus biofilms and, in combination with common anti staphylococcal antimicrobials, effectively killed bacterial cells being released from the biofilm. These fibrinolytic agents were not cytotoxic and did not affect the host immune response. The rat model of infection successfully demonstrated the activity of the selected fibrinolytic agents alone and in combination with antimicrobials on established biofilms in vivo TrypLE and nattokinase most successfully removed adherent cells from plasma-coated surfaces and significantly improved the efficacy of existing antimicrobials against S. aureus biofilms in vitro and in vivo "[39].

Zapotoczna M, etal: "High-level resistance to antimicrobial drugs is a major factor in the pathogenesis of chronic Staphylococcus aureus biofilm-associated, medical devicerelated infections. Antimicrobial susceptibility analysis revealed that biofilms grown for $\leq 24$ hours on biomaterials conditioned with human plasma under venous shear in ironfree cell culture medium were significantly more susceptible to anti staphylococcal antibiotics. Biofilms formed under these physiologically relevant conditions were regulated by SaeRS and dependent on coagulase-catalyzed conversion of fibrinogen into fibrin. In contrast, SarA-regulated biofilms formed on uncoated polystyrene in nutrient-rich bacteriological medium were mediated by the previously characterized biofilm factors poly- $\mathrm{N}$-acetyl glucosamine, fibronectin-binding proteins, or autolytic activity and were antibiotic resistant. Coagulase-mediated biofilms exhibited increased antimicrobial resistance over time ( $>48$ hours) but were always susceptible to dispersal by the fibrinolytic enzymes plasmin or nattokinase. Biofilms recovered from infected central venous catheters in a rat model of devicerelated infection were dispersed by nattokinase, supporting the important role of the biofilm phenotype and identifying a potentially new therapeutic approach with antimicrobials and fibrinolysis drugs, particularly during the early stages of device-related infection"[40].

de Almeida J, et al: "Disruption of the matrix of endodontic biofilms will aid in their removal from a root canal. The aim of this study was to investigate the efficacy of EDTA and a modified salt solution (MSS) to detach bacteria from biofilms.

Forty-eight-hour-old Enterococcus faecalis biofilms were grown on glass coverslips and then treated for 1 hour by immersion in 17\% EDTA or MSS. Phosphate-buffered saline served as a negative control. Then, residual biofilm cells on the substrate and the detached cells in the supernatant were collected. Viability was verified by the colony-forming unit (CFU) counting method. Propidium monoazide (PMA) treatment in conjunction with quantitative polymerase chain reaction (qPCR) was also performed to detect the presence of E. faecalis $16 \mathrm{~S}$ ribonucleic RNA genes. Data were analyzed using 1-way analysis of variance and Tukey or Kruskal-Wallis and Dunn tests. The Pearson R test evaluated the correlation between results from CFU and PMA $(\alpha=5 \%)$.

qPCR showed that EDTA detached 99\% of biofilm cells, and MSS detached $94 \%$ of biofilm cells (both $P<.001$ ). In contrast to EDTA, MSS was highly antimicrobial. The treatment promoted an ample log 7 reduction of the attached cells $(P<.001)$, and almost no live cells were detected in the supernatant $(P<.001)$. Positive correlations between CFU and qPCR with PMA were observed ( $r=0.959$ and $r=0.729)$.

EDTA detached cells in biofilms with a minor antimicrobial effect. Besides a great antimicrobial effect, MSS also detached biofilm cells. These dispersals of biofilms give insights into new endodontic biofilm removal strategies "[41].

Choi YS, et al: "Removal of bacterial biofilm from the root canal system is essential for the management of endodontic disease. Here we evaluated the antibacterial effect of $\mathrm{N}$-acetyl cysteine (NAC), a potent antioxidant and mucolytic agent, against mature multispecies endodontic biofilms consisting of Actinomyces naeslundii, Lactobacillus salivarius, Streptococcus mutans and Enterococcus faecalis on sterile human dentin blocks. The biofilms were exposed to NAC $(25,50$ and $100 \mathrm{mg} / \mathrm{mL})$, saturated calcium hydroxide or $2 \%$ chlorhexidine solution for 7 days, then examined by scanning electron microscopy. The biofilm viability was measured by viable cell counts and ATP-bioluminescence assay. NAC showed greater efficacy in biofilm cell removal and killing than the other root canal medicaments. $100 \mathrm{mg} /$ $\mathrm{mL}$ NAC disrupted the mature multispecies endodontic biofilms completely" [42].

Dinicola S, et al: "Biofilms are microbial communities consisting of bacteria, extremely capable to self-reproduce on biological surfaces, causing infections. Frequently, these biofilms are resistant to classical antibacterial treatments and host immune response. New adjuvant molecules are mandatory in clinical practice. N-acetyl cysteine (NAC), a precursor to the antioxidant glutathione, has been investigated for its effectiveness both in inhibiting biofilm formation and in destroying developed biofilms. The aim of our study was to conduct a systematic literature review of clinical trials involving NAC as adjuvant treatment to eradicate pre-formed mature biofilms and to inhibit new biofilm production.

A careful analysis of the Medline was conducted and eight studies were selected according to the following criteria: site of infection, kind of bacteria, design of the research, dose of the treatment, administration, biological effects and results. We fixed an arbitrary scale of scores from 0 (lowest score) to 5 (highest score) for each criterion and a threshold value of 3 . The studies analyzed, with score over 3 , suggested a potential role for NAC as adjuvant molecule in 
the treatment of bacterial biofilms, with an excellent safety and efficacy profile. NAC, in combination with different antibiotics, significantly promoted their permeability to the deepest layers of the biofilm, overcoming the problem of the resistance to the classic antibacterial therapeutic approach. these results are encouraging to a more widespread clinical use of NAC, as adjuvant therapy for microbial infections followed by biofilm settle, which may occur in several body districts, such as the vaginal cavity [43].

Ham Y, et al: "Biofilm provides a bacterial hiding place by forming a physical barrier and causing physiological changes in cells. The elimination of biofilm is the main goal of hygiene. Chemicals that are inhibitory to biofilm formation have been developed for use in food, personal hygiene products, and medical instruments. Mono acylglycerols are recognized as safe and are used in food as emulsifiers. In this study, the inhibitory activity of mono acylglycerols on bacterial biofilm formation was evaluated systematically with four bacterial strains, Aeromonas hydrophila, Streptococcus mutans, Xanthomonas oryzae, and Yersinia enterocolitica. Mono acylglycerols with two specific lengths of fatty acid moiety, monolaurin and monobehenin, were found to have strong inhibitory activity toward bacterial biofilm formation of S. mutans, $X$. oryzae, and $Y$. enterocolitica in a strain specific manner. this result suggested that biofilm formation was not inhibited by the detergent characteristics of monoacylglycerols. This suggestion was supported by the inhibitory action of monolaurin on biofilm development but not on the initial cell attachment of Y. enterocolitica in flow cytometric observation. it was also suggested that two distinct response mechanisms to monoacylglycerols existed in bacteria "[44].

Richter K, et al: "In light of emerging multi-drug-resistant bacteria, alternative strategies to antibiotics are emerging. The present study evaluated the activity of colloidal silver nanoparticles (AgNPs) of different shapes against biofilms formed by Staphylococcus aureus (SA), methicillin-resistant SA (MRSA), and Pseudomonas aeruginosa (PA). Colloidal quasi-spherical, cubic, and star-shaped AgNPs were synthesized, and their cytotoxicity on macrophages (THP1) and bronchial epithelial cells (Nuli-1) was analyzed by the lactate dehydrogenase assay. The antibiofilm activity was assessed in vitro by the resazurin assay and in an in vivo infection model in Caenorhabditis elegans. Cubic and starshaped AgNPs induced cytotoxicity, while quasi-spherical AgNPs were not toxic. Quasi-spherical AgNPs showed substantial antibiofilm activity in vitro with $96 \%( \pm 2 \%)$, $97 \%( \pm 1 \%)$, and $98 \%$ ( $\pm 1 \%$ ) biofilm killing of SA, MRSA, and PA, respectively, while significantly reducing mortality of infected nematodes. The in vivo antibiofilm activity was linked to the accumulation of AgNPs in the intestinal tract of C.elegans as observed by 3D X-ray tomography. Quasispherical AgNPs were physically stable in suspension for over 6 months with no observed loss in antibiofilm activity.
While toxicity and stability limited the utilization of cubic and star-shaped AgNPs, quasi-spherical AgNPs could be rapidly synthesized, were stable and nontoxic, and showed substantial in vitro and in vivo activity against clinically relevant biofilms. Quasi-spherical AgNPs hold potential as pharmacotherapy as topical treatment for biofilm-related infections "[45].

Gujie Mi, et al: "In recent years, the use of nanoparticles, such as liposomes, polymersomes, and solid lipid nanoparticles, as delivery systems for these conventional antibiotics has achieved considerable success in overcoming antibiotic resistance and reducing toxic sides effects both in vitro and in vivo. Several of these nanoparticle formulations have progressed into clinical investigations.

Arikace developed by Insmed is a liposomal formulation of amikacin targeting non tuberculous mycobacterial(NTM) Lung Disease. It has been shown to improve tolerability, safety and efficacy of the encapsulated antibiotics and is currently in a phase 3 study. The improvements in antibiotictreatment using these nanoparticle-based delivery systems are presumably due to enhanced drug stability and improved pharmacokinetic profiles. Linhaliq (formerly known as Pulmaquin)is another example of antibiotic (ciprofloxacin) liposomal formulation that targets P.aeruginosa infections in cysticfibrosis patients and is now in application for market approval. Almost all clinical candidates so far have been limited to liposomal formulations. This is most likely due to the higher maturity and the considerable success achievedin cancer treatments with liposomal systems. Future investigations should also focus on other systems such as polymersomes and solid lipid nanoparticles to take the full advantage of their unique physicochemical properties. It is also worth noting that delivery of antibiotics through nanoparticles does not change their underlying molecular targets. Bacteria are thought to quickly develop resistance to these reformulated antibiotics. Metal and metal oxide nanoparticles, such as AgNPs, offer an appealing alternative as they typically exert antibacterial activity by targeting both cell walls and intracellular targets simultaneously Adv. Healthcare via physical interactions, ion release and generation of ROS, making it harder for bacteria to develop resistance.

Chen, et al. made it possible to generate poly elemental metallic nanoparticles with precise size and composition. This will not only allow us to systematically evaluate how binary, ternary, or quaternary metallic nanoparticles interact with bacteria, but will also allow us to harvest synergistic effects among multiple metallic elements to maximize antibacterial efficiency. the exact antimicrobial mechanisms of most metal and metal oxide nanoparticles remain elusive. Toxicities of many of these NPs have been a concern and have to be properly addressed using physiological relevant animal models and eventually in clinical studies. This could be particularly useful in mitigating concerns over chronic 
toxicities. With respect to device associated infections, bio inspired nanostructured surfaces represent a promising strategy to combat biofilm formation without the use of antimicrobials. As such, there is minimal concern for the development of antibiotic resistance. The interactions of bacteria with these surfaces are not fully understood. Much of our knowledge so far is overwhelming from in vitro and in silico experimentation.

In vivo experimentations involving nanostructured surfaces without the use of antimicrobials is scarce and is much needed to fully understand the interactions between bacteria and surfaces in a much more complex environment that contains not only a plethora of physiochemical cues but also host factors. The ability to readily fabricate sophisticated structures over larger surfaces may also limit its broad application.

Future investigations should focus on further understanding how bacteria interact with surfaces and how these interactions may contribute to bacterial colonization, which could be fueled by cutting-edge imaging techniques and advanced computational modeling. a better understanding of how nanoparticles and nanostructured surfaces interact with bacteria through technological innovations in both materials sciences and biology will help guide future designs of more effective systems to combat the growing problem of antibiotic resistance and biofilm-related device infections "[46].

Sousa C, et al: "nanoscience and nanotechnology can be applied into several fields including medicine, materials science and security. On the case of medical applications, it can go from the development of biosensors to the improvement of biomaterials with increased biocompability or even with antimicrobial properties. Although, of all advantages of nanotechnology, several concerns have been raised, namely the high surface reactivity and the ability of nanoparticles to cross cell membranes that in some cases can be beneficial, like in cancer treatment, but it may also have negative health and environmental impacts. This type of technology can also be employed to study microbial cells at the nanoscale level, using e.g. atomic force microscopy (AFM), which allows the study in situ of membrane proteins and live cells at high resolution. It is also possible to apply this technique to determine, for instance, adhesion forces, or to map surface properties and receptor sites on cells, as well to measure cellular interactions at the single-cell and single-molecule levels. As mentioned previously, the single cell interaction is the first step for the adhesion and biofilm formation and, using this approach, it is possible to analyze step by step the formation of a biofilm and understand the initial phenomenon. Concerning biomedical biofilms, nanotechnology is emerging as one of the most auspicious methodologies for its prevention and control. The main nano approaches that have demonstrated the most promising results include: silver nanoparticles, drug delivery nanocarriers or phage therapy. Silver nanoparticles
Antimicrobial activity of silver, copper and other metal ions is well known and, of all the elements, silver has been described as the one with the highest levels of toxicity for microorganisms and the lowest toxicity for animal cells. this metal has a broad antimicrobial activity spectrum against both Gram+, and Grambacteria, as well as yeasts. On the case of bacteria it is known that inhibits replication by binding to the microbial DNA and it also switches off important enzymes, leading to microbial death. The nanoscale materials have recently appeared as new antimicrobial agents due to their high surface area to volume ratio and unique chemical and physical properties. Silver nanoparticles (NPs), which are clusters of silver atoms, exhibit very strong bactericidal activity against both Gram + and Gram- bacteria, including multi resistant strains.

According to the literature, the silver NPs have affinity for protein aceous compounds, where they combine with $\mathrm{SH}$ groups inducing protein denaturation and corresponding enzyme inactivation. On a model for Saccharomyces cerevisiae it was demonstrated that a nanosilver based treatment induces the formation of clusters at the cell wall periphery composed by silver and sulphur, with significant levels of phosphorus, showing a specific reactivity of silver species to phosphorus-containing compounds suggested that the carbonyl groups from amino acid residues of proteins are able to bind to metal and, additionally, Ag+ ions interact with amide groups with a preferential binding to amide carbonyl oxygen. This may cause disarrangement on the secondary structures of proteins, indicating the presence of inactive conformations. Given the increasing microbial resistance and consequent development of resistant strains to traditional antimicrobial agents, silver NPs or Nano coatings constitute nowadays an important antimicrobial agent with numerous applications in medicine. Indeed, silver NPs have not been shown to cause bacterial resistance, which is presumably due to the fact that, unlike antibiotics, silver NPs do not exert their antibacterial effects only in a particular site but at several degrees such as bacterial wall, protein synthesis and DNA. These nano silver coatings exert their antimicrobial properties in vivo by slowly releasing Ag+ ions. The considerable surface-to-volume ratio of the NPs enables a constant local supply of $\mathrm{Ag}+$ ions at the coating-tissue interface and also allows an improved contact with the (Ed) microorganism. As a result, prevention of microorganisms adhesion and biofilm formation is more prolonged than in other antimicrobial approaches involving silver ions or metallic silver impregnation. The advantage of impregnation of medical devices with silver NPs is that it protects both outer and inner surfaces of devices and there is continuous release of silver ions providing antimicrobial activity. Nanosilver, as particles, coating, or even impregnated on the medical device are thus emerging as a next-generation of antimicrobial agents.

Some studies have raised some concerns regarding silver NPs biosafety, there are studies demonstrating the efficacy 
of silver NPs in reducing or preventing biofilm formation on catheter-materials both in vitro and in animal models. Studies with patients are still scarce.

Novel drug delivery carrier systems even though the efficacy of nano coatings and silver NPs to prevent biofilm formation on catheters, there is an important limitation: the ability of the material to adsorb always the same concentration of the drug and also the ability to control their release, which in most cases results in a non-controlled elution of the drug in the first hours subsequent to the insertion.

Drug delivery has been a subject of intense studies over the recent years. The objective is to accomplish sustained (or slow) and/or controlled drug release and improve efficacy, safety, and/or patient comfort. These new drug delivery carriers can be considered as a way to prevent the colonization and biofilm formation, and the most exploited ones for elimination of microbial biofilms on biomedical devices are lipid- and polymer-based carrier systems Liposomes as drug carriers.

In 1961, Alec D Bangham, firstly described the liposomes at the Babraham Institute, in Cambridge. Liposomes are artificially prepared vesicles made of lipid bilayer that can be used as drug carriers. They are appealing drug carrier systems, especially against colonizing microorganisms, due to several factors such as: good biocompatibility; they are able to carry drugs with very different characteristics (from hydrophobic to hydrophilic drugs, e.g); the encapsulation of the drug protects it from the biological milieu; and also allows the drug to be transported to a specific target site. Several studies have been performed concerning the interaction between liposomes and bacterial biofilms.

Halwani, et al. showed that this new strategy can be used to deliver two agents at the same time in order to prevent P.aeruginosa biofilm formation and resistance in vitro. Di Tizio, et al. developed a strategy of ciprofloxacin delivery consisting of a liposomal hydrogel that reduced bacterial adhesion to silicone catheter in a rat model of persistent $P$. aeruginosa peritonitis.

This technique opened new perspectives for the development of new antimicrobial peritoneal dialysis catheters or as well other types of catheters. Buckler, et al. reported that liposomal antifungal lock therapy can be considered as a possible alternative to catheter removal.

In fact, this therapy was previously tested with success in an animal model of C.albicans biofilm-associated CVC infection. A different study using liposomal amphotericin B (LAMB) at the minimal inhibitory concentration in a catheter continuous flow model for Candida showed that after $24 \mathrm{~h}$ treatment with LAMB, the growth of hyphae diminished in $20 \%$ in comparison with the traditional antifungal therapy; additionally, the thickness of extracellular matrix was imperceptible. In 2008, eleven liposomal delivery systems were approved for clinical used, being two of them against fungi (Abelcet and Ambisone). So, the use of liposomes as drug carriers seems to be advantageous over other therapies used to prevent biofilm formation on biomedical surfaces. Liposomes can target matrix or biofilm by specific attachment, allowing the drug to be released in the vicinity of the microorganisms, although in the case of yeast cells adhesion to human cells there is the need to deepen the knowledge regarding the ability of this systems to prevent the adhesion but not to affect adhered cells.

Polymer carriers for the past years, the use of biocompatible or biodegradable polymers has gained importance on the medical field. Polymer carriers from both natural and synthetic origins, have several advantages over other types of drug nanocarriers, such as the liposomes which can be taken up by macrophages, and accumulate in the liver and spleen, even when the liposomes are coated with polyethyleneglycol (PEG). The use of polymers as drug carriers for antimicrobial agents, associated to implants infections, can be an extremely valuable alternative.

Among these products, polymeric microspheres, polymer micelles, and hydrogel-type materials have been shown to be effective nanocarriers in enhancing drug targeting specificity, lowering systemic drug toxicity, improving treatment absorption rates, and providing protection for the pharmaceuticals against biochemical degradation. These polymer drug delivery systems are based on "nanocarriers" which are formed by mixing polymeric chemical compounds with drugs forming complex and large molecules, which "carry" the drug across physiological barriers. Poly(rhyleneglycol)-poly(alpha, beta-asparic acid), carboxylates, and hetero bifunctional polyethylene glycol, constitute examples of these polymeric compounds. Another advantage of this system is the possibility to add a pore forming polymer, which will increase the amount of drug loaded on the carrier, as described by Ruggeri, et al. which developed an antimicrobial polyurethane system containing two antibiotics, cefamandole nafate and rifampin and PEG as a pore forming polymer. Crisante, et al. developed a nanostructured polymer system for antibiotic delivery using bovine serum albumin or polyallylamine as pore forming substances.

de Prijck, et al. showed that polydimethyl siloxane (PDMS) can be mixed with different antifungal agents to induce reduction on Candida biofilm formation. Using a similar strategy described for bacterial biofilms, Donelli, et al. used polyurethane loaded with fluconazole and pore-former agents in order to promote the release of the antifungal drug.

The entrapment of an antifungal drug in the polymer of PEG as a pore former significantly improved fluconazole release, while the use of polymer with a higher molecular weight, as porogen albumin resulted in a tighter controlled drug release with an improved antifungal activity over time. 
In order to diminish Candida-associated denture stomatitis, which is a serious clinical problem, a similar nanotechnological approach was tested: antifungal drugs, such as miconazole, were incorporated in polymethacrylic acid (PMAA) which can be covalently bound onto diurethane dimethacrylate, a denture resin. This system was very effective, as the PMAA-resin discs drug-containing were sustained for a prolonged period of time (weeks and months). The combination of antimicrobial drugs in polymers with pore formers leads to a controlled release over time, which is a promising approach in the development of medical devices.

Phage therapy: The use of bacteriophages (phages) has been another strategy studied for the prevention and control of biomedical biofilms. Considering the increasing apprehension with antimicrobial resistance in hospitals worldwide nowadays, there is a renewed interest in phage therapy. Phage therapy has been successfully used to treat experimental infections, including bloodstream infections and meningitis, in poultry and animals, and antimicrobialresistant infectious diseases in humans such as dysentery, skin infections, lung infections, meningitis, wound infections and osteomyelitis, caused by a range of organisms including Staphylococcus, Streptococcus, Escherichia coli, P.aeruginosa, Shigella and Salmonella. The use of phages to control infections caused by biofilms has advantages over treatment with other conventional antimicrobial agents, namely by the fact that phages have a very strong bactericidal activity and can replicate at the site of infection.

Doolittle and coll, described, in 1996, a progeny phage which propagates radially throughout a biofilm, suggesting that a single dose of phage could treat a biofilm infection. The progeny phage infects adjacent cells and degrades the biofilm matrix. Besides, it was demonstrated that some phages are able to produce enzymes (depolymerises) that hydrolyse and degrade EPS matrix of a biofilm. Curtin and Donlan, demonstrated, using an in vitro model system, that a phage active against S.epidermidis could be incorporated into a hydrogel coating on a catheter and significantly reduce biofilm formation on its surfaces. Fu, et al. studied, in vitro, the effect of pre-treating hydrogel-coated catheters with P.aeruginosa phages on biofilm formation, and observed a significant reduction in the number of biofilm cells. This shows that the combination of two nanotechnological approaches can improve the final outcome: the control of biofilm formation on indwelling devices. despite these results, some aspects still must be considered prior to the use of phage therapy in humans, such as, bacterial resistance to phage, inactivation by the patient's immune system, impure phage preparations that could contain endotoxins or phageencoded virulence genes that can incorporate into the host bacterial genome. The use of phage mixtures or engineered phages can provide successful strategies to overcome these problems" [47].
Sara M Soto, "Antimicrobial Treatment of Biofilms: Treatment of biofilm-associated infections is a field that requires further study, in part due to the high levels of antibiotic resistance exhibited by biofilm structures conferred in part by the exo polysaccharide matrix. Several studies recommend combination therapy as the treatment of choice in biofilmassociated infections, with macrolides being one of the first antibiotics chosen. Macrolides (erythromycin, clarithromycin, and azithromycin) present high "in vitro" and "in vivo" antibiofilm activity against biofilm-associated infections caused by Gram-bacteria inhibiting the production of a key component of the matrix, alginate. Macrolides have been shown to be effective against P.aeruginosa, another Gram- bacteria, and more recently against Staphylococcus spp. biofilms. This antibiofilm activity was first described "in vitro" exposing $P$. aeruginosa sessile cells to clarithromycin and erythromycin. The antibiotic combination, clarithromycin plus vancomycin, demonstrated the ability to eradicate both biofilm and planktonic cells as well as to eradicate biofilm on the titanium washers used in animal experiments. Roxithromycin plus imipenem favor a higher penetration of neutrophils into biofilm structure destabilizing the biofilm. Conversely, macrolides have been shown to enhance biofilm formation in Gram + bacteria due to an increase in the expression of biofilm-related genes such as icaA, atlE, fruA, pyrR, sarA, and sigB. This fact has an important clinical implication because the macrolide levels needed for enhancing biofilm formation could be found in clinical niches or settings.

Another approach using antimicrobials consists in coating and impregnating the catheters with these antimicrobial agents. The aim of this procedure is to avoid bacterial attachment to the catheter surface and the posterior development of biofilm. In this sense, the silver has also been used to coat catheters because it has bactericidal actions. Silver has broad-spectrum antimicrobial activity. The antimicrobial action of silver compounds is proportional to the bioactive silver ion released and its availability to interact with bacterial or fungal cell membranes. It has been observed that silver alloy coating prevents adherence and the growth of biofilm-embedded bacteria by $50 \%$. Synthetic cationic peptide variants derived from natural peptides have been used as strategy to target biofilm. Some substances showing antibacterial properties, such as gendine (gentian violet plus chlorhexidine), nitrous oxide, and nitrofurazone (nitrofuran), have been used to modify the surface of urinary catheters. the risk of using antibiotics to treat the catheter surface may lead to the development of antimicrobial resistance when the drug levels become sub inhibitory.

\section{New trends in antibiofilm treatments}

Catheters coated with hydrogels or antibiotics: Hydrogels are cross-linked, insoluble, hydrophilic polymers that trap water. This characteristic provides the catheter with an increase in surface lubrication which consequently decreases 
the bacterial adhesion to this surface and demonstrates a role in the reduction of encrustation of catheters. The ability of these hydrogels to prevent CAUT remains unclear. Kazmierska, et al. observed that a hydrogel layer increased the aggregation of planktonic cells, causing an increase in nucleated crystals, provoking more rapid catheter blockage in comparison with the uncoated silicone. This negative effect was suppressed when active agents were added to the hydrogel.

Silver alloy has been used in hydrogel-coated urinary catheter observing a decrease of up to $45 \%$ of CAUTI. Minocycline-rifampicin-coated catheters have been shown to inhibit the biofilm formation of Gram+ and Gram- pathogens, except P.aeruginosa and Candida spp. one problem with this may be the possible development of resistant phenotypes among the bacteria. no silver-resistant mutants were collected in the aforementioned studies. A nanoparticle is a microscopic particle with a dimension of less than $100 \mathrm{~nm}$. These particles have the capacity to attach and penetrate into bacterial cells, disrupt the bacterial membrane, and interact with chromosomal DNA.

Nanoparticles of MgF have been used for coating glass surfaces observing an inhibition of biofilm formation by both, E. coli and S. aureus. Catheters have also been coated with these nanoparticles and a significant reduction of bacterial colonization was observed over a period of 1 week in comparison with the catheter uncoated catheter control. This group also demonstrated the antibacterial and antibiofilm activity of yttrium fluoride (YF3) nanoparticles which showed low solubility and provided extended protection. In addition, another advantage of these nanoparticles was their low cytotoxicity. Microwave irradiated $\mathrm{CaO}$ nanoparticles (CaO-NPs) have also shown the potential to inhibit biofilm formation against Gram-and Gram+ bacteria. Silver nanoparticles have also been used for impregnating medical devices due to the silver antimicrobial properties previously commented in this review. These nanoparticles have been used in medical and pharmaceutical nano engineering applied to the delivery of therapeutic agents, diagnostic approaches, and as part of biosensors.

Several studies have demonstrated the "in vivo" and "in vitro" inhibition of biofilm formation by numerous bacterial species and using determined nanoparticle concentrations. The mechanism of action of silver nanoparticles remains unknown. Another aspect related to silver nanoparticles is the toxicity to eukaryotic cells which remains uncharacterized. Iontophoresis is a physical process in which ions flow diffusively in a medium driven by an applied electric field. This method enhances the efficacy of antibiofilm agents "in vitro". It has been observed that low electrical currents enhance the activity of tobramycin and biocides against P.aeruginosa biofilm. This effect has only been observed among those antibiotics that are effective against planktonic cells. Iontophoresis has also been studied to prevent biofilm formation and encrustation by P.mirabilis showing that the application of electric current to these catheters fitted with silver electrodes significantly decreased their encrustation. Nevertheless, "in vivo" studies have yet to be performed in this respect.

Enzyme Inhibitors: Urease, the enzyme that allows $P$. mirabilis to hydrolyze urea to ammonium ions, has been an important target in the study of new antibiofilm compounds. In this sense, fluorofamide has been a candidate molecule because it is able to prevent the increase in $\mathrm{pH}$ by Proteus mirabilis "in vitro", thereby inhibiting the formation of urea crystal and the subsequent encrustation and catheter obstruction. Other natural compounds, such as vanillic acid, natural plum juice, and germa- $\gamma$-lactones, among others, presented the ability to strongly inhibit bacterial growth as well as the formation of crystals in catheters by the inhibition of the urease enzyme.

Lu and Collins, generated a bacteriophage which expressed a biofilm-degrading enzyme during infection. The enzyme associated with the bacteriophage was DspB and it is produced by one species of Actinobacillus. DspB hydrolyses a crucial adhesion needed for biofilm formation and integrity in both E. coli and Staphylococcus and attacks the bacterial cells in the biofilm and the biofilm matrix simultaneously. The percentage of eradication using this bacteriophage-enzyme combination was about $99.9 \%$.

The second messenger, c-di-GMP, has been studied in depth because it is highly conserved among bacterial species, being an important candidate for studies on biofilm inhibition. c-di-GMP is synthetized via diguanylate cyclases (DGC). Inhibition of DGC activity leads to a reduction in biofilm formation by a decrease in the intracellular levels of c-di-GMP. Several molecules have been shown to inhibit biofilm formation on urinary catheters by P.aeruginosa via an inhibition on the DGC WspR enzyme and they have the ability to disperse formed biofilm of P.aeruginosa and A.baumannii. These small molecules are denominated LP 3134, LP 3145 , LP 4010, and LP 1062.

The use of liposomes could be one method for improving the effectiveness of antibiotics. They act as carriers of drug molecules, increasing their length of life and reducing toxicity in the host. Liposome-encapsulated antimicrobial agents have been successfully tested in humans and in animal models, being directed to eradicate protozoa, fungal, and bacterial infections. Liposomes can be applied in the eradication of formed biofilm because when the antibiotic is encapsulated in a liposome carrier it does not interact with the EPS, improving its antibiofilm effect, and it is protected from degradation by antibiotic-inactivating enzymes (such as $\beta$-lactamases) which can appear in the biofilm matrix. Thus, liposomal containing tobramycin and bismuth reportedly 
penetrated and killed bacteria in the core of the P.aeruginosa biofilms, in contrast to both free compounds that only killed and detached bacteria from the biofilm surface. Liposomes containing clarithromycin have been tested against biofilmforming P.aeruginosa, observing a significant reduction in biofilm production as well as in bacterial twitching, swarming, and swimming motility. In addition, clarithromycinentrapped liposomes showed less cytotoxicity than the free drug. In spite of the excellent results obtained, more studies are needed in CAUTI and other biofilm-related infections.

\section{Bacterial Interference}

This method is related to the antagonism between different bacteria species during the colonization of surfaces and biofilm formation. Briefly, the colonization of a surface by nonpathogenic bacteria could prevent the adherence of pathogenic bacteria thereby avoiding infection. Several avirulent strains of $E$. coli have been used as a method to reduce urinary catheter colonization by a wide variety of pathogens. Thus, the E. coli HU2117 strain, derived from E. coli 83972, that causes persistent colonization without symptomatic infection, has been used for coating urinary catheters, observing a reduction of biofilm formation by other pathogens. This strain presented a deletion of the papG gene resulting in a lack of $\mathrm{P}$-fimbriae. The E. coli 83972 strain has also been used for coating urinary catheters, demonstrating a reduction in the development of UTIs in persons undergoing an intermittent catheterization programme. A study performed among catheterized patients with spinal cord injury who were inoculated with a nonpathogenic E. coli strain in the bladder showed that these patients had less probability of developing an episode of UTI during the oneyear follow-up period.

Bacteriophages are the natural predators of bacteria. They are viruses that specifically infect bacteria. Among them, lytic phages are able to disrupt the normal bacterial metabolism, favoring viral replication. Phages have been used for treating some infectious diseases in humans mainly related to S.aureus due to their bactericidal activity. The phage characteristics that allow them to control biofilm are the capacity to replicate at the site of infection, the production of enzymes (depolymerases) that degrade the EPS of the biofilm matrix, and their capacity to propagate through the biofilm. These phages have been incorporated into hydrogel-coating catheters, and a reduction has been observed in biofilm formation by Staphylococcus epidermidis and P.aeruginosa. In addition, the use of lytic bacteriophages against established biofilm of P.mirabilis and E. coli caused a reduction of three to four log cycles. These lytic phages also prevented biofilm formation on catheters coated with hydrogel containing bacteriophages. The reduction of formation observed was about $90 \%$.

Quorum sensing (QS) is a cell-density-dependent chemical signaling system that allows individual cells to release small signal molecules to the surroundings to make their presence known. The small signal molecules are known as auto inducers and coordinate cell-density-dependent gene expression. QS is used to coordinate gene expression and regulate numerous processes that are involved in virulence such as motility and biofilm formation being necessary for planktonic bacteria to adopt the biofilm phenotype.

An efficient quorum sensing inhibitor (QSI) should have the following characteristics: (1) have a low molecular mass able to inhibit the expression of genes related to QS, (2) be highly specific for the QS-regulators, (3) not show toxicity to the eukaryotic hosts, (4) not interfere with the basal metabolic processes of bacteria in order to avoid the development of resistances, (5) be chemically stable, resistant to host metabolism, and able to reside in the host for a sufficient long time.

Several QSI have been identified to date, many having been isolated from nature. the pyrimidinone compound inhibits biofilm formation and disrupts and removes the biofilm deposited. HSL analogues are compounds analogous to the QS signal and compete with the native signals for binding to the receptor, blocking QS, and inhibiting biofilm formation in Saureus. Garlic extract was found to enhance the susceptibility to tobramycin by altering the architecture of the bacterial biofilms. Peptides also show QSI activity. Thus, the RNAIII inhibiting peptide was able to inhibit agrmediated biofilm formation in drug resistant $\mathrm{S}$. epidermidis.

\section{Low-energy surface acoustic waves}

It has been demonstrated that surface acoustic waves (SAW) interfere with adhesion of planktonic microorganisms to cellular surfaces. SAW reduces biofilm bioburden on catheter segments in suspensions with several Gram-and Gram+ bacteria as well as fungi indicating its efficacy against a broad spectrum of microorganisms. Power intensities of 0.1 and $0.2 \mathrm{~mW} / \mathrm{cm} 2$, generating vibration frequencies of $95 \mathrm{KHz}$ and $220 \mathrm{kHz}$ with acoustic pressure amplitudes of 0.1 and $0.22 \mathrm{kPa}$, respectively, were the conditions used in the "in vitro" experiments on avoiding adhesion.

The efficacy of this approach was studied in a rabbit model, and it was observed that catheter-associated vibrating activators were found to maintain urine sterile for 7 days, whereas control catheters (without vibrations) resulted in bacteriuria within 1.5 days. This effect was observed against E. coli, Enterococcus faecalis, Candida albicans, and P.mirabilis.

\section{Antiadhesion agents}

The prophylaxis of UTIs using anti adhesive compounds/ molecules is currently an important objective in clinical research. The main characteristic of an anti-adhesive compound is that it should specifically interact with the adhesins of the pathogen, inhibiting the union between 
pathogen and eukaryotic cell. These anti adhesive compounds cause a decrease in invasion or infection of host epithelial cells, also avoiding recurrence. One of the compounds most frequently studied is cranberry extract. The anti-adherence effect of cranberry against uropathogenic E. coli (UPEC) is due to the presence of A-type proanthocyanidin trimmers in the cranberry extract that acts as an antiadhesion agent. Other antiadhesion agents are those denominated mannosides, curlicides, and pilicides. These agents inhibit the biogenesis of adhesins required for biofilm formation and adhesion to epithelial cells. The most studied agents are the mannosides that inhibit FimH attachment to host receptors. FimH is the tip of the type 1 fimbriae of $E$. coli that mediates the first step in biofilm formation. Mannosides seem to have a good prophylactic role in UTIs caused by E. coli since they not only interfered with adherence but also enhance the effect of the antimicrobial agent cotrimoxazol. In this sense, nanodiamond particles, covertly modified with mannose moieties, are able to efficiently inhibit $E$. coli type 1 fimbriae-mediated adhesion to eukaryotic cells.

Salicylate is a member of a large group of pharmaceuticals referred to as nonsteroidal anti-inflammatories and it is the active component of the analgesic aspirin. Salicylate has shown to decrease biofilm formation of UPEC inhibiting type 1 fimbriae expression. In addition, the effect of salicylate in biofilm reduction could be intensified by the decrease of OmpA expression that causes a decrease in the extracellular matrix.

Bacterial biofilms seem to be reservoir for molecules that act as antagonists of bacterial adhesion. An example is a polysaccharide produce by E. coli that excludes S.aureus from mixed E. coli and S.aureus biofilms. the possible patient outcomes related to biofilm-related infections make the indepth search for compounds with antibiofilm properties necessary in order to prevent the formation of and/or disrupt the development of biofilm. The ideal situation is a compound or a group of compounds that present not only antibiofilm activity but also the capacity to eradicate multi-drug-resistant bacteria. It is also important to study the antibiofilm activity of new combinations of therapeutic strategies including new ones and old antibiotics. Virulence factors should be considered important targets for developing antivirulence compounds that may be applied in the prevention and treatment of infectious diseases "[48].

Braj R Singh, et al: "Quorum sensing (QS) is a chemical communication process that Pseudomonas aeruginosa uses to regulate virulence and biofilm formation. Disabling of QS is an emerging approach for combating its pathogenicity. Silver nanoparticles (AgNPs) have been widely applied as antimicrobial agents against human pathogenic bacteria and fungi, but not for the attenuation of bacterial QS. Here wemycofabricated AgNPs (mfAgNPs) using metabolites of soil fungus Rhizopus arrhizus BRS-07 and tested their effect on QS-regulated virulence and biofilm formation of P.aeruginosa. Transcriptional studies demonstrated that mfAgNPs reduced the levels of LasIR-RhlIR. Treatment of mfAgNPs inhibited biofilm formation, production of several virulence factors (e.g. LasA protease, LasBelastrase, pyocyanin, pyoverdin, pyochelin, rhamnolipid, and alginate) and reduced AHLs production.

Further genes quantification analyses revealed that mfAgNPs significantly down-regulated QS regulated genes, specifically those encoded to the secretion of virulence factors. The results clearly indicated the anti-virulence property of mfAgNPs by inhibiting P.aeruginosa QS signaling. Excessive and indiscriminate uses of antibiotics have led to the emergence of multi-drug resistant (MDR) Pseudomonas aeruginosa, a notorious opportunistic pathogenic bacterium causing infect "[49].

Kaiser SJ, et al: "The antimicrobial properties of natural isothio cyanides (ITCs) found in plants such as nasturtium (Tropaeolum majus) and horseradish (Armoracia rusticana), and the need of new chemotherapeutic options for treatment of infections caused by multi-drug-resistant and biofilm forming Gram negative bacteria such as P.seudomonas aeruginosa $(\mathrm{Pa})$, led us to evaluate the effects of three major ITCs, allylisothiocyanate (AITC), benzylisothiocyanate (BITC), and phenylethyl-isothiocyanate (PEITC), and a mixture (ITCM) adapted to the ITC composition after release of active components out of natural sources.

Out of $105 \mathrm{~Pa}$ isolates 27 isolates with increased biofilm formation were selected for testing. The effects of ITCs on $\mathrm{Pa}$ were evaluated regarding (1) planktonic bacterial proliferation, (2) biofilm formation, (3) metabolic activity in mature biofilms, and (4) synergism of ITCs and antibiotics.

(1) Each ITC had anti-Pa activity. Mean minimum inhibitory concentrations (MICs) were $(\mu \mathrm{g} / \mathrm{ml}$, mean \pm standard deviation): AITC $103 \pm 6.9$; BITC, $2145 \pm 249$; PEITC 29,423 \pm 1652 ; and ITCM, $140 \pm 5$. (2). Treating bacteria with PEITC and ITCM in concentrations below the MIC significantly inhibited biofilm formation. Particularly, ITCM reduced biofilm mass and bacterial proliferation. (3) ITCs significantly inhibited metabolic activity in mature biofilms. (4). Combining ITCs with meropenem synergistically increased antimicrobial efficacy on Pa biofilms. ITCs represent a promising group of natural anti-infective compounds with activity against Pa biofilms" [50].

$\mathrm{Fu}$ B, et al: New antimicrobial agents are urgently needed to treat infections caused by drug-resistant pathogens and by pathogens capable of persisting in biofilms. The aim of this study was to identify traditional chinese herbs that could inhibit biofilm formation of P.seudomonas aeruginosa, an important human pathogen that causes serious and difficultto-treat infections in humans. A luxCDABE-based reporter system was constructed to monitor the expression of six key 
biofilm-associated genes in P.aeruginosa. The reporters were used to screen a library of 36 herb extracts for inhibitory properties against these genes. The results obtained indicated that the extract of Herba patriniae displayed significant inhibitory effect on almost all of these biofilm-associated genes. Quantitative analysis showed that H.patriniae extract was able to significantly reduce the biofilm formation and dramatically altered the structure of the mature biofilms of P.aeruginosa. Further studies showed H.patriniae extract decreased exopolysaccharide production by P.aeruginosa and promoted its swarming motility, two features disparately associated with biofilm formation. These results provided a potential mechanism for the use of H.patriniae to treat bacterial infections by traditional chinese medicines and revealed a promising candidate for exploration of new drugs against P.aeruginosa biofilm-associated infection" [51].

Kumar L, et al: "Quorum sensing in Pseudomonas aeruginosa plays an imperative role in virulence factor, biofilm formation and antimicrobial resistance. Blocking quorum sensing pathways are viewed as viable anti-virulent therapy in association with traditional antimicrobial therapy. Anti-quorum sensing dietary phytochemicals with may prove to be a safe and viable choice as anti-virulent drug candidates. Previously, our lab proved zingerone as potent anti-biofilm agenthence; furtheritsanti-virulentand anti-quorumactivities were evaluated. Zingerone, besides decreasing swimming, swarming and twitching phenotypes of P.aeruginosa PA01, reduced biofilm forming capacity and production of virulence factors including rhamnolipid, elastase, protease, pyocyanin, cell free and cell bound hemolysin $(p<0.001)$ indicating anti-virulent property attributing towards attenuation of virulence of $P$. aeruginosa. Further zingerone not only had marked effect on the production of quorum sensing signal molecules by clinical isolates of P.aeruginosa but also showed significant interference with the activation of QS reporter strains. To study the mechanism of blocking quorum sensing cascade, in silico analysis was carried out. Anti-QS activity was attributed to interference with the ligand receptor interaction of zingerone with QS receptors (TraR, LasR, RhlR and PqsR). Zingerone showed a good comparative docking score to respective auto inducer molecules which was even higher than that of vanillin, a proven anti-quorum sensing phytochemical. The results of the present study revealed the anti-quorum sensing activity of zingerone targeting ligandreceptor nteraction, hence proposing zingerone as a suitable anti-virulent drug candidate against P.aeruginosa infections “[52].

Farias KS, et al: "Essential oils, mixtures of volatile compounds, are targets of research for new antimicrobial drugs. In order to verify the potential from species of the Nectandra genus, the present study evaluated the essential oils from Nectandra amazonum, Nectandra cuspidata, Nectandra gardineri, Nectandra hihua and Nectandra megapotamica to prospect samples with high concentration of a component and its antibacterial, antibiofilm and anti-Trichomonas vaginalis activities. The essential oils from the leaves and barks were extracted by steam distillation and analyzed by gas chromatography coupled to mass spectrometry (GC-MS). The concentrations of 10 and $100 \mu \mathrm{g} / \mathrm{mL}$ of the essential oil were evaluated and the inhibition of bacterial growth and biofilm formation were measured, while for the evaluation of anti-T. vaginalis trophozoite viability, the concentrations from 7.8 to $1000 \mu \mathrm{g} / \mathrm{mL}$ were tested. Seventy-three compounds were identified from essential oils, highlighted bicyclogermacrene (up to $49.9 \%$ ), elemi in (up to $42.4 \%$ ), intermodal (up to $58.2 \%$ ), (E)-asarone (up to $45.9 \%$ ) and (+)- $\alpha$-bisabolol (up to $93.7 \%$ ). The essential oil from N. megapotamica leaves presented $93.7 \%$ of (+)- $\alpha$-bisabolol and demonstrated the high capacity of inhibition of the biofilm formation, in particular, against Staphylococcus aureus methicillin resistant (MRSA) and Pseudomonas aeruginosa. This sample also had significant activity against T. vaginalis (IC50 of $98.7 \mu \mathrm{g} / \mathrm{mL}$ ) and demonstrated cytotoxic and hemolytic effects in vitro cells and human erythrocytes. In general, the Nectandra genus revealed high chemical variability and a N.megapotamica specimen accumulated a compound on high concentration with great potential for biotechnological exploration as a new antibiofilm and anti-T. vaginalis" [53].

Tse-Kai $\mathrm{Fu}$, et al: "More than $80 \%$ of infectious bacteria form biofilm, which is a bacterial cell community surrounded by secreted polysaccharides, proteins and glycolipids. Such bacterial superstructure increases resistance to antimicrobials and host defenses. Thus, to control these biofilm-forming pathogenic bacteria requires antimicrobial agents with novel mechanisms or properties. P.seudomonasa eruginosa, a Gram- opportunistic nosocomial pathogen, is a model strain to study biofilm development and correlation between biofilm formation and infection. In this study, a recombinan themolymph plasma lectin (rHPLOE) cloned from Taiwanese Tachypleus tridentatus was expressed inan E. coli system. This rHPLOE was shown to have the following properties: (1) Binding to P.aeruginosa PA14 biofilm through a unique molecular interaction with rhamnose-containing moietieson bacteria, leading to reduction of extracellular di-rhamnolipid (a biofilm regulator); (2) decreasing downstream quorum sensing factors, and inhibiting biofilm formation; (3) dispersing the maturebiofilm of P.aeruginosa PA14 to improve the ecacies of antibiotics; (4) reducing $P$. aeruginosa PA14cytotoxicity to human lung epithelial cells in vitro and (5) inhibiting P.aeruginosa PA14 infection of zebra fish embryos in vivo. Taken together, rHPLOE serves as an anti-biofilm agent with a novelmechanism of recognizing rhamnose moieties in lipopolysaccharides, di-rhamnolipid and structuralpolysaccharides (Psl) in biofilms. Thus rHPLOE links glycan-recognition to novel anti-biofilmstrategies against pathogenic bacteria" [54].

Lokender Kumar, et al: "Enterococcus faecalis is a major opportunistic pathogen that readily forms protective biofilms 
leading to chronic infections. Biofilms protect bacteria from detergent solutions, antimicrobial agents, environmental stress, and effectively make bacteria 10 to 1000 -fold more resistant to antibiotic treatment. Extracellular proteins and polysaccharides are primary components of biofilms and play a key role in cell survival, microbial persistence, cellular interaction, and maturation of E. faecalis biofilms. Degradation of biofilm components by mammalian proteases is an effective antibiofilm strategy because proteases are known to degrade bacterial proteins leading to bacterial cell lysis and growth inhibition. Here, we show that human matrix metalloprotease- 1 inhibits and disrupts E. faecalis biofilms. MMPs are cell-secreted zinc- and calcium-dependent proteases that degrade and regulate various structural components of the extracellular matrix. Human MMP1 is known to degrade type- 1 collagen and can also cleave a wide range of substrates. We found that recombinant human MMP1 significantly inhibited and disrupted biofilms of vancomycin sensitive and vancomycin resistant E. faecalis strains. The mechanism of antibiofilm activity is speculated to be linked with bacterial growth inhibition and degradation of biofilm matrix proteins by MMP1. These findings suggest that human MMP1 can potentially be used as a potent antibiofilm agent against E. faecalis biofilms “[55].

Yucui Liu, et al: "Streptococcus mutans has been reported as a primary cariogenic pathogen associated with dental caries. The bacteria can produce glucosyltransferases (Gtfs) to synthesize extracellular polysaccharides (EPSs) that are known as virulence factors for adherence and formation of biofilms. an ideal inhibitor for dental caries is one that can inhibit planktonic bacteria growth and prevent biofilm formation. Bergenia crassifolia (L.), widely used as a folk medicine and tea beverage, has been reported to have a variety of bioactivities. The present study aimed to explore the effect of B. crassifolia (L.) leaf extracts on the biofilm of Streptococcus mutans. The B. crassifolia (L.) leaf extracts showed inhibitory effects by decreasing viability of bacteria within the biofilm, as evidenced by the XTT assay, live/dead staining assay and LDH activity assay, and could decrease the adherence property of S. mutans through inhibiting Gtfs to synthesize EPSs. In addition, the reduced quantity of EPSs and the inhibition of Gtfs were positively correlated with concentrations of test samples. Finally, the MTT assay showed that the extracts had no cytotoxicity against normal oral cells. the extracts and sub-extracts of B. crassifolia leaves were found to be antimicrobial and could reduce EPS synthesis by inhibiting activities of Gtfs to prevent bacterial adhesion and biofilm formation. B. crassifolia leaves have potential to be developed as a drug to prevent and cure dental caries "[56].

Deep G1, et: "Prostate cancer (PCA) is the leading malignancy in men and the second leading cause of cancerrelated deaths. Hypoxia (low $\mathrm{O} 2$ condition) is considered an early event in prostate carcinogenesis associated with an aggressive phenotype. In fact, clinically, hypoxia and hypoxia-related biomarkers are associated with treatment failure and disease progression. Hypoxia-inducible factor 1 (HIF-1) is the key factor that is activated under hypoxia, and mediates adaptation of cells to hypoxic conditions through regulating the expression of genes associated with angiogenesis, epithelial-to-mesenchymal transition (EMT), metastasis, survival, proliferation, metabolism, sternness, hormone-refractory progression, and therapeutic resistance. Besides HIF-1, several other signaling pathways including PI3K/Akt/mTOR, NADPH oxidase (NOX), Wnt/b-catenin, and Hedgehog are activated in cancer cells under hypoxic conditions, and also contribute in hypoxia-induced biological effects in HIF-1-dependent and -independent manners. Hypoxic cancer cells cause extensive changes in the tumor microenvironment both local and distant, and recent studies have provided ample evidence supporting the crucial role of nanosized vesicles "exosomes" in mediating hypoxiainduced tumor microenvironment remodeling Exosomes' role has been reported in hypoxia-induced angiogenesis, sternness, activation of cancer-associated fibroblasts (CAFs), and EMT. existing literature suggests that hypoxia plays a predominant role in PCA growth and progression, and PCA could be effectively prevented and treated via targeting hypoxia/hypoxia-related signaling pathways"[57].

Massimo Ammirante, et al: "Prostate cancer often responds to hormone ablation therapy or chemotherapy by becoming more aggressiveand metastatic. Bcellsrecruited into hormonedeprived tumors by C-X-C motif chemokine 13 (CXCL13) play an important role in this process. We investigated how androgen ablation induces CXCL13 expression and found that CXCL13 is expressed by myofibroblasts within the tumor microenvironment that become activated as a result of low oxygen tension and hypoxia in androgen-deprived tumors. Hypoxia activates hypoxia-inducible factor 1 (HIF1) and induces TGF- $\beta$ expression, which converts fibroblasts to myofibroblasts and stimulates CXCL13 production. We show that several treatments that block CXCL13 expression, including immunodepletion of myo fibroblasts, blockade of TGF- $\beta$ signaling, and phosphodiesterase-5 (PDE5) inhibitors, inhibit B-cell recruitment into androgen-deprived prostate tumors and prevent the emergence of a more aggressive type of cancer"[58].

Gustafsson MV, et. Al: "In addition to controlling a switch to glycolytic metabolism and induction of erythropoiesis and angiogenesis, hypoxia promotes the undifferentiated cell state in various stem and precursor cell populations. Here, we show that the latter process requires Notch signaling. Hypoxia blocks neuronal and myogenic differentiation in a Notch-dependent manner. Hypoxia activates Notchresponsive promoters and increases expression of Notch direct downstream genes. The Notch intracellular domain interacts with HIF-1alpha, a global regulator of oxygen 
homeostasis, and HIF-1alpha is recruited to Notch-responsive promoters upon Notch activation under hypoxic conditions. Taken together, these data provide.

Cao Y1, Ma J; Increasing evidence suggested obesity, measured by body mass index (BMI), was associated with prostatecancer-specificmortality, anditsimpactonbiochemical recurrence was also inconclusive. We systematically searched MEDLINE, EMBASE, and bibliographies of retrieved studies up to January 5, 2010. We used random-effects meta-analysis to assess the relative risks (RR) of prostate cancer-specific mortality and biochemical recurrence associated with a 5 $\mathrm{kg} / \mathrm{m}(2)$ increase in BMI. Among the six population-based cohort studies in 1,263,483 initially cancer-free men, 6,817 prostate cancer deaths occurred; a $5 \mathrm{~kg} / \mathrm{m}(2)$ increase in BMI was associated with 15\% (RR: 1.15, 95\% confidence interval (CI): 1.06-1.25, $P<0.01$ )higher risk of dying of prostate cancer. In the six post diagnosis survival studies on 18,203 patients with 932 prostate cancer deaths, a $5 \mathrm{~kg} / \mathrm{m}(2)$ increase in BMI was associated with $20 \%$ higher prostate cancer-specific mortality (RR: $1.20,95 \% \mathrm{CI}: 0.99-1.46, P=$ $0.06)$. In the sixteen studies which followed 26,479 prostate cancer patients after primary treatment, a $5 \mathrm{~kg} / \mathrm{m}(2)$ increase in BMI was significantly associated with $21 \%$ increased risk of biochemical recurrence (RR: $1.21,95 \% \mathrm{CI}$ : 1.11-1.31 $P$ $<0.01$ ). Elevated BMI is associated with risk of prostate cancer-specific mortality in prospective cohort studies and biochemical recurrence in prostate cancer patients. Its association with prostate cancer-specific mortality in diagnosed patients' needs to be further evaluated" [60].

Timothy Stark, et al: "Inflammation as a biological process is typically associated with infection, but it can be triggered by autoimmune diseases, allergies, and tissue trauma. Cytokines released by inflammatory cells activate signal cascades providing a growth advantage for surrounding tumors. Many cytokine functions and selecting and interactions can be manipulated to promote growthChronic inflammation has been proven as an integral contributor to cancer ontogeny. Dissecting the relationship between prostate cancer evolution and the process of inflammation is an ongoing pursuit. Current observation and experimentation clearly demonstrate that inflammation is functionally synergistic with high-grade aggressive prostate tumors and ultimately metastatic spread $(7,19)$. The evidence-based knowledge so far supports the regulatory role of the inflammatory cascade in prostate cancer growth and progression to metastasis by exerting a tight control on the tumor microenvironment landscape, the ECM and EMT cycling outcomes. Inflammatory cells within the tumor microenvironment promote angiogenesis by releasing a number of growth factors, including VEGF, IL-8, and bFGF during tumorigenic growth (Figure 1). The release of soluble inflammatory mediators such as TNF- $\alpha$, TGF- $\beta$, IL7, IL-2, RANTES, and macrophage inflammatory protein-1b activates surrounding stromal cells and provokes dramatic remodeling of the ECM (10). Further inflammatory cytokines released in the setting of reactive stroma, actuate the cellular response towards induction of EMT. Indeed, the multiplicity of signaling factors influencing inflammation in the prostate microenvironment and the complexity of biological events linking inflammation to prostate cancer progression and metastasis provide a promising platform for potential therapeutic targeting. To date, numerous investigative efforts have exploited the anti-tumor efficacy of non-steroidal antiinflammatory agents, modulators of inflammatory cytokine and transcription factor activation, epigenetic modification of the EMT phenotypic program dictating cell polarity and invasiveness and repression of TAM signaling "[61].

DSW Benoit, et al: "Although seemingly disparatedisease, treatment of solid tumors and biofilm-derived infections face a common problem: drugs often fail to reach and kill cancer cell and microbial pathogen because of local microenvironment heterogeneities.

Tissue hereteogeneity and compartmentalization alter accessibility of chemotherapic agents, provides protection from exogenoulsy introduced antibiotics and promotes drugs resistance. Altered immunoresponces are often found within tumor and biofilm microenvironment" [62].

Guillaume Lambert, et al: "Both bacterial and tumor cells can evade death induced by exposure to drugs through various mechanisms.

The easiest strategy is to move to an environment that contains a lower concentration of a cytocidal agent. This is achieved through swimming by bacterial cells and through metastasis by tumor cells. Alternatively, the cell population can create a milieu where the drug has limited access to the cells. This has been demonstrated to be a function of biofilms in a bacterial colony and a function of an altered tumor microenvironment (including the vasculature) for tumor. Cells. One of the most intriguing methods of evading death in both cell populations depends on a probabilistic phenotypic switching mechanism. In this situation, a small fraction of the bacterial or tumor cell population is in a state that is not responsive to the cytotoxic properties of the drug. This has been called 'a persisted phenotype' for bacterial communities and has recently been described as a mechanism whereby tumour cells can escape death caused by exposure to drugs".

Biofilm and tumor stroma One of the physiological responses of bacteria to external stress is to assemble into a biofilm. Pseudomonas aeruginosa is often used as a model of biofilm development; in culture, they produce an exopolymer matrix that protects cells from surrounding environmental stresses. The formation of a biofilm greatly increases the resistance of a population to a hostile environment by shielding cells, for example, from antibiotics. Biofilms, however, limit the influx of nutrients and oxygen owing to 
the decreased diffusion of chemicals through the biofilm matrix. Although bacterial cells trigger the expression of fermentative pathways in the absence of oxygen33, this metabolic pathway creates endogenous oxidative stress within the exo polymer matrix, which in turn increases the mutation rate of the cells.

Cancer is not just a collection of cells replicating and evolving uncontrollably; it is an ecosystem cells surrounding a tumor (such as fibroblasts, immune cells and endothelial cells) are part of a tumor tissue and co-evolve with cancer cells. For instance, stromal cells such as fibroblasts associated with cancerous tissues increase extracellular matrix (ECM) production. Similarly to bacterial biofilms, the increased matrix deposition not only reduces the effectiveness of chemotherapeutic drugs to penetrate a tumor but also reduces the amount of oxygen and nutrients reaching the centre of a tumor. Analogously to biofilms, tumor cells may also switch to a fermentative pathway when oxygen is unavailable: anaerobic glycolysis allows cells to produce ATP but inadvertently leads to the acidification of the tumor microenvironment through the release and fermentation of lactate" [63].

Chris Kresser: "Clinicians instead need to break down the biofilm, attack the pathogenic bacteria within, and mop up the leftover matrix, DNA, and minerals. Biofilm disruptors are the first course of action. Enzymes such as nattokinase and lumbrokinase have been used extensively as coatings on implants to fight biofilms. Promising enzymes include proteases, plasmin, and streptokinase. Mucolytic enzyme $\mathrm{N}$-acetyl cysteine (NAC) is a precursor of glutathione and an antioxidant. Effective against biofilms on prosthetic devices, in vitro biofilms, and chronic respiratory infections: NAC is recognized as a "powerful molecule" against biofilms. Lauricidin (other forms: monolaurin, lauric acid, and glycerol monolaurate) is a natural surfactant found in coconut oil that helps inhibit the development of biofilms. Colloidal silver is effective at treating topical biofilms, such as in wound dressings. Applications in vivo are still under research (Figures 4,5).

Klaire Labs Inter Fase Plus and Kirkman Biofilm Defense, two commercial products formulated to effectively disrupt biofilm. Mixtures of herbal antimicrobials can be effective: like, berberine, artemisinin, citrus seed extract black walnut hulls, Artemisia herb, Echinacea, goldenseal, gentian, fumitory, galbanum oil oregano oil. Once the biofilm is destabilized and microbes are treated, binders help clean up the mess.

EDTA disrupts biofilms and also chelates minerals in the matrix.

Chitosan and citrus pectin are two other options".

Experimental project hypothesis.

In order to verify on field the amount of reduction of antibiotics in chronic prostatitis is proposed to implement a daily diary of patient physical activity, behavior, sitting position and other to be correlated to the Number of relapses of prostatitis event and to the amount of antibiotics used in a time object of observation (Figures 6,7).

The same also a correlation between the effective antibiofilm strategies must be correlated to the amount of replaspes.

\section{Discussion}

After this reviews is possible to say that the pathological movens in some form of chronic prostatitis conditions can be considered as function of different facts: quantitative consideration (time $\mathrm{x}$ kind of stressing condition, by month, antibiofilm specific treatment):

In natural position: prolonged time sitting: time of exposure, Body weight, Trauma: bicycle and other traumatic sports.

Amount of sports activity related pelvic physiology, Congestion pelvic- prostatic: venous, lymphatic, IPB condition.

\section{The lymphatic drainage of pelvis}

Sacral lymph node

- locate along middle sacral a. and lateral sacral a.

- Receive lymph from posterior pelvic wall, rectum,prostate or uterus

Common iliac lymph node

- Lie along common iliac artery

- Receive afferents from all the above nodes

- Efferents pass to lumbar lymph node

Dept of Anatomy SDV

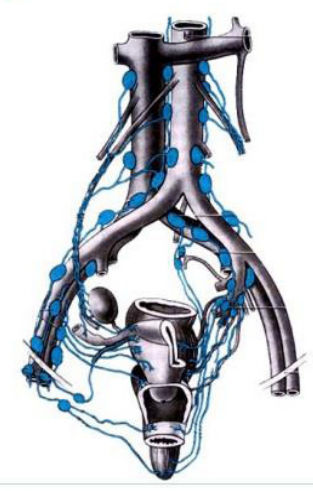

Figure 4: The lymphatic drainage of pelvis.

Blood supply of Prostate Gland

- Arterial supply:

Branches of Internal Iliac artery

- Branches from inferior

vesical artery

- Branches from middle recta artery

- Branches from Internal pudendal artery

Venous drainage Veins form prostatic plexus Drain into internal iliac vein

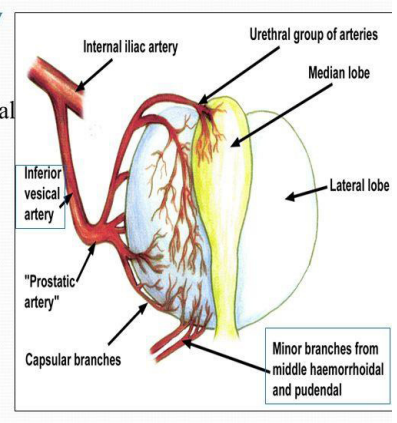

Figure 5: Blood supply of prostate Gland. 
Fibrotics changes: after flogotic events, IPB, AGEING, Ageing (IPB), Flogotic reactions, Micro calcifications.

Immune infiltration after acute fact: macrophages, Lymphocites, Bacteria (mono-polimicrobial), biofilm Dietary irritants (food, drinks, alcohol) and other Antibiotics:

Profile of prostatic penetration, spectrum of activity, posology, cicle, duration of therapy, resistances, MDR, drug classes.

Oral or parenteral antibiotics sub ministration, associations, toxicity Biofilm agents associations (see new strategy) whit antibiotics (biofilm agents artificial, natural, fitoterapic, physical methods). Antiflammatory topics, systemics, fans, steroids, Antiedemigen, Antihormonal: finasteride, dutasteride and other Sildenafil 5PDE inhibitors and analogue Fitotherapic. SERENOA R and other remedies.

Alfa blokers other (biofeedback, pelvic muscle relaxant exercise, tepid water). An objective daily register of lifestyle is unuseful instruments that can contribute to reduce antibiotics overuse in prostatic condition.

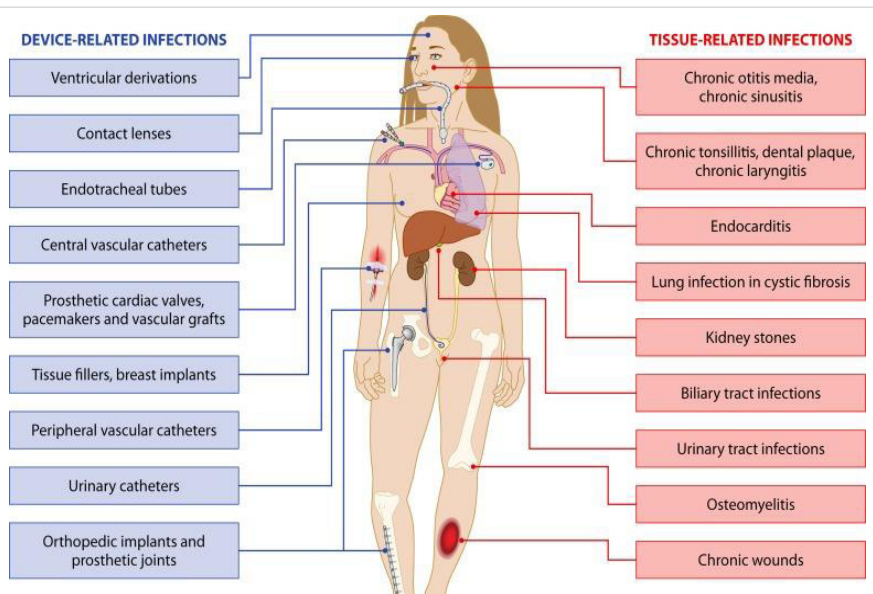

Figure 6: Image From article for Scintific Purpose: Biofilm-Related Infections: Bridging the gap between Clinical Management and Fundamental Aspects of Recalcitrance toward Antibiotics. David Lebeaux, a,b Jean-Marc Ghigo,a Christophe Beloina.

David Lebeaux,a,b Jean-Marc Ghigo,a Christophe Beloina

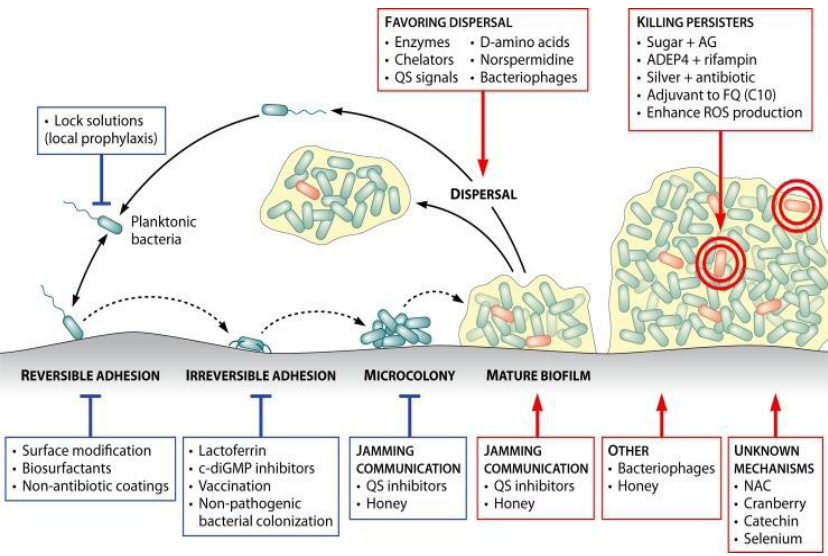

Figure 7: Antibiofilm strategies arising from fundamental research. Approaches to preventing formation of biofilms are depicted in blue; approaches to eradicating an established biofilm are shown in red.
A quantitative relationship between objective lifestyle behavior and number of relapses or antibiotics use can be crucial to translate to the patients the real meaning that simply change in behavior can improve a lot their quality of life.

And related biofilm problems: A relationship:

Global therapeutic efficacy biofilm related infectious:

$\mathrm{GTE}=\mathrm{A} \times \mathrm{B} \times \mathrm{P}$

( $A=$ antibiotic-antimicrobial activity, $\mathrm{b}=$ antibiofim agent activity, $p=$ persitence of local action).

If $\mathrm{B}=10 \%-70 \%$ in example surely the global effect will be less the $100 \%$ with relapses, and higher amount of antibiotics used.

\section{Conclusion}

After this review work Is possible to say that an $100 \mathrm{~kg}$ weight Taxi Driver ( 8 hour of sitting by day) working present the same probability in relapses in chronic prostatitis of an athletic $70 \mathrm{~kg}$ of weight with a standing position of work?

The same what effect produce 1-2 hour of bicycle? versus walking for the same time?

To reduce antibiotic abuse in chronic prostatitis is needed to measure the amount of stressing condition that can aggravate this pathology. (daily diary of activity), and to transfer to patient correct and incorrect lifestyle.

Decongestionant measure can improve this situation and also a periodic "washing procedure strategy - detox " of prostatic tissue from bacteria, biofilm, white cells - flogosis mediators and other products may be an use full instrument to prevent relapses in chronic prostatitis. (a clearance of toxic-pathologic movens: Into prostate tissue flux and out from this.

The focus must not only be the antibiotics molecule but first the way to reduce biofilm if present and to balance other pathological factors like congestive condition. (and also in prostatic cancer biofilm role must be strongly considered).

To observe the progression of research related other biofilm - human infectious make possible to find new strategy also in chronic prostatitis biofilm related. (See the medical devices like endoprotesis).

In order to improve clinical efficacy in therapy of chronic prostatitis with high rate of relapsis Is strongly needed to transfer knowledge obtained from other field like Medical Devices Antibiofilm strategy also in urologic field (chronic prostatitis), pharmaceutical- pharmacological, pharmaceutical techniques, molecular biology, physical techniques, biological instruments, local site persistent penetration, associations toxicological aspects and others like dynamics, kinetics, drug delivery, nano drug strategies, liposomes and others under research. 
The same a better understanding of prostatic biofilm phenomena and its implications due to a creation of a new micro-environment whit drugs resistance is an interesting model also for prostatic cancer progression and therapy complete knowledge.

A last consideration: why until today we not have drugs specifically registered with first indication officially approved: Biofilm reduction?

\section{Clarification}

This article is produced under a pharmacological- and pharmaceutical point of view, without any therapeutic or diagnostic intent only to produce new research hypotesys to be submitted to the researcher.

\section{References}

1. Kogan MI, Matsionis AE, Belousov II, Povilaitite PE. Morphological evidence of the ischemic nature of the prostatic fibrosis in the classical chronic pelvic pain syndrome / IIIB chronic prostatitis. Urologiia. 2018; 3: 12-19.

PubMed: https://www.ncbi.nlm.nih.gov/pubmed/30035413

2. Vaidyanathan R, Vibhash C. Mishra. Chronic prostatitis: Current concepts. Indian J Urol. 2008; 24: 22-27.

PubMed: https://www.ncbi.nlm.nih.gov/pubmed/19468353

3. Well-Being, Money SA. Therapeutic Devices Should be implemented in the Healthcare System for the Treatment of Chronic Noncancerous Prostate and Kidney Diseases Saving People's. Ann Mil Health Sci Res. 2018; 16: e81033.

4. Kogan MI, Belousov II, Bolotskov AS. Arterial blood flow in the prostate in the syndrome of chronic pelvic pain/chronic prostatitis. Urologiia. 2011; 3: 22-28.

PubMed: https://www.ncbi.nlm.nih.gov/pubmed/21874666

5. Kim HJ, Park JW, Cho YS, Cho CH, Kim JS, et al. Pathogenic role of HIF1 ? in prostate hyperplasia in the presence of chronic inflammation. Biochim Biophys Acta. 2013; 1832: 183-194.

PubMed: https://www.ncbi.nlm.nih.gov/pubmed/22986049

6. Portia $\mathrm{T}$, Jing-Hua $\mathrm{Y}$, Yedan $\mathrm{Li}$, Lori BL, Kazem M. Structural modifications of the prostate in hypoxia, oxidative stress, and chronic ischemia. Korean J Urol. 2015; 56: 187-196.

PubMed: https://www.ncbi.nlm.nih.gov/pubmed/25763122

7. Kimio S, Saori N, Katsumi K, Katsuhiro A, Tomoyuki U, et al. Laboratory investigation Pelvic venous congestion with castration causes chronic prostatitisin rats. Int J Urol. 2016; 23: 431-435.

8. Timothy J Coker, Daniel M Dierfeldt. Acute Bacterial Prostatitis: Diagnosis and Management. Am Fam Physician. 2016; 93: 114-120. PubMed: https://www.ncbi.nlm.nih.gov/pubmed/26926407

9. Shazia R Chaudhry, Khalid Chaudhry. Anatomy, Abdomen and Pelvis. Pelvis. 2019

10. Chiappino G, Pisani E. Prostate diseases of occupational origin. Med Lav. 2002; 93: 67-72.

PubMed: https://www.ncbi.nlm.nih.gov/pubmed/12087801

11. Konstantinos S, Vittorio M, Gianpaolo P, Nektaria R, Richard L, et al. Gram-positive microorganisms isolated during Chronic Bacterial Prostatitis investigation. Hellenic Urology. 2019; 30: 35-49.

12. Azadzoi KM, Babayan RK, Kozlowski R, Siroky MB. Chronic Ischemia Increases Prostatic Smooth Muscle Contraction in the Rabbit. J Urol.
2003; 170: 659-663.

PubMed: https://www.ncbi.nlm.nih.gov/pubmed/12853851

13. Berger AP, Deibl M, Leonhartsberger N, Bektic J, Horninger W, et al. Vascular damage as a risk factor for benign prostatic hyperplasia and erectile dysfunction. BJU Int. 2005; 96: 1073-1078.

PubMed: https://www.ncbi.nlm.nih.gov/pubmed/16225531

14. Chen $X$, Hu C, Peng Y, Lu J, Yang NQ, et al. Association of diet and lifestyle with chronic prostatitis/chronic pelvic pain syndrome and pain severity: a case-control study. Prostate Cancer Prostatic Dis. 2016; 19: 92-99.

PubMed: https://www.ncbi.nlm.nih.gov/pubmed/26666410

15. Andrew PB, Michel P, Richard EB, Dean AT. Management of Men Diagnosed With Chronic Prostatitis/Chronic Pelvic Pain Syndrome Who Have Failed Traditional Management. Rev Urol. 2007; 9: 63-72. PubMed: https://www.ncbi.nlm.nih.gov/pmc/articles/PMC1892625/

16. Gao DJ, Guo YS, Yu HY, Wang YJ, Cui WG. Prevalence and related factors of prostatitis-like symptoms in young men; Zhonghua Nan Ke Xue. 2007; 13: 1087-1090.

PubMed: https://www.ncbi.nlm.nih.gov/pubmed/18284056

17. Taylor BC, Noorbaloochi S, McNaughton-Collins M, Saigal CS, Sohn MW, et al. Excessive Antibiotic Utilization in Men with Prostatitis. Am J Med. 2008; 121: 444-449.

PubMed: https://www.ncbi.nlm.nih.gov/pubmed/18456041

18. Jinjin MA, Gharaee-Kermani M, Lakshmi K, John MH, Jeremy A, et al. Prostatic Fibrosis is Associated with Lower Urinary Tract Symptoms. J Urol. 2012; 188: 1375-1381.

PubMed: https://www.ncbi.nlm.nih.gov/pubmed/22906651

19. Niemitz $C$. The evolution of the upright posture and gait-a review and a new synthesis. Naturwissenschaften. 2010; 97: 241-263. PubMed: https://www.ncbi.nlm.nih.gov/pubmed/20127307

20. Ran Z, Andrea KC, Jordan DD, Edward LG, Walter CW, et al. Physical Activity and Chronic Prostatitis/Chronic Pelvic Pain Syndrome. Med Sci Sports Exerc. 2015; 47: 757-764.

PubMed: https://www.ncbi.nlm.nih.gov/pubmed/25116086

21. Dikov D, Bachurska S, Staikov D, Sarafian V. Intraepithelial lymphocytes in relation to NIH category IV prostatitis in autopsy prostate. Prostate. 2015; 75: 1074-1084.

PubMed: https://www.ncbi.nlm.nih.gov/pubmed/25917232

22. Anim JT, Udo C, John B. Characterisation of inflammatory cells in benign prostatic hyperplasia. Acta Histochem. 1998; 100: 439-449. PubMed: https://www.ncbi.nlm.nih.gov/pubmed/9842422

23. Pavone C, Caldarera E, Liberti P, Miceli V, Di Trapani D. Prostate Diseases Correlation between Chronic Prostatitis Syndrome and Pelvic Venous Disease; A Survey of 2,554 Urologic Outpatients. European Urology. 37: 400-403.

24. Luisetto M, Naseer A, Ghulam RM, Ahmed YR, Farhan AK, et al. Endogenous Archeological Sciences: Anatomy, Physiology, Neuroscience, Biochemistry, Immunology, Pharmacology, Oncology, Genetics as Instrument for A New Field of Investigation? Modern Global Aspects for a New Discipline Open Access J Addict \& Psychol. 2018; 1.

25. Levine JA. Sick of sitting. Diabetologia. 2015; 58: 1751-1758.

26. Hadi D, Alireza C, Haleh G, Mehran K. Adverse Effects of Prolonged Sitting Behavior on the General Health of Office Workers. J Lifestyle Med. 2017; 7: 69-75.

PubMed: https://www.ncbi.nlm.nih.gov/pubmed/29026727

27. Loran OB, Dunaevski I, Vishnevski AE, Vashkevich VI. The joint use of hyperbaric oxygenation and EHF therapy in benign prostatic hyperplasia and its combination with chronic prostatitis. Urol Nefrol 
(Mosk). 1997; 2: 32-34

PubMed: https://www.ncbi.nlm.nih.gov/pubmed/9206881

28. Motoaki S, Panagiota T, Ryo O, Shogo S, Masashi H, et al. Prostatic ischemia induces ventral prostatic hyperplasia in the SHR; possible mechanism of development of BPH. Asian J Urol. 2017; 4: 158-163.

29. Motoaki S, Panagiota T, Shogo S, Takehiro S, Yukako K, et al. Prostatic ischemia induces ventral prostatic hyperplasia in the SHR; possible mechanism of development of BPH. Sci Rep. 2014; 4: 3822. PubMed: https://www.ncbi.nlm.nih.gov/pubmed/24448152

30. Jason G, Omar S, Noel LS, Gunjan J, Sohrab V, et al. Clinical utility of hyperbaric oxygen therapy in genitourinary medicine. Med Gas Res. 2018; 8: 29-33.

PubMed: https://www.ncbi.nlm.nih.gov/pubmed/29770194

31. Declan J McKenna, Rachel E, Klaus P. Current challenges and opportunities in treating hypoxic prostate tumors. J Cancer Metastasis Treat. 2018; 4:11.

32. Ficarra V. Editor's Choice is chronic prostatic inflammation a new target in the medical therapy of lower urinary tract symptoms (LUTS) due to benign prostate hyperplasia (BPH)? BJU International. 2013; 112: 421-422.

PubMed: https://www.ncbi.nlm.nih.gov/pubmed/23879899

33. Andrzej G, Joczyk-Matysiak E, Marzanna S, Ryszard M, et al. Phage Therapy in Prostatitis: Recent prospects. Front Microbiol. 2018; 9:1434. PubMed: https://www.ncbi.nlm.nih.gov/pubmed/30008710

34. Bartoletti R, Cai T, Nesi G, Albanese S, Meacci F, et al . The impact of biofilm-producing bacteria on chronic bacterial prostatitis treatment: results from a longitudinal cohort study. World JUrol. 2014;32: 737-742. PubMed: https://www.ncbi.nlm.nih.gov/pubmed/23918259

35. Corvec S, Furustrand Tafin U, Betrisey B, Borens O, Trampuz A. Activities of fosfomycin, tigecycline, colistin, and gentamicin against extended-spectrum-?-lactamase-producing Escherichia coli in a foreign-body infection model. Antimicrob Agents Chemother. 2013; 57: 1421-1427.

PubMed: https://www.ncbi.nlm.nih.gov/pubmed/23295934

36. David L, Jean-Marc G. Biofilm-Related Infections: Bridging the Gap between Clinical Management and Fundamental Aspects of Recalcitrance toward Antibiotics. Microbiol Mol Biol Rev. 2014; 78: 510-543

PubMed: https://www.ncbi.nlm.nih.gov/pubmed/25184564

37. Muhamad AB, Judy McKimm, Seraj Zohurul $H$, Anwarul AM, Mainul $\mathrm{H}$. Chronic tonsillitis and biofilms: a brief overview of treatment modalities. J Inflammation Res. 2018; 11: 329-337.

PubMed: https://www.ncbi.nlm.nih.gov/pubmed/30233227

38. Batoni G, Maisetta G, Esin S. Antimicrobial peptides and their interaction with biofilms of medically relevant bacteria. Biochim Biophys Acta. 2016; 1858: 1044-1060.

PubMed: https://www.ncbi.nlm.nih.gov/pubmed/26525663

39. Hogan S, O'Gara JP, O'Neill E. Novel Treatment of Staphylococcus aureus Device-Related Infections Using Fibrinolytic Agents. Antimicrob Agents Chemother. 2018; 62.

PubMed: https://www.ncbi.nlm.nih.gov/pubmed/29203484

40. Zapotoczna M, McCarthy H, Rudkin JK, O'Gara JP, O'Neill E. Coagulase in Staphylococcus aureus Biofilm Development Reveals New Therapeutic Possibilities for Device-Related. J Infect Dis. 2015; 212: 1883-1893.

PubMed: https://www.ncbi.nlm.nih.gov/pubmed/26044292

41. De Almeida J, Hoogenkamp M, Felippe WT, Crielaard W, van der Waal SV. Effectiveness of EDTA and Modified Salt Solution to Detach and Kill Cells from Enterococcus faecalis Biofilm. J Endod. 2016; 42: 320-323. PubMed: https://www.ncbi.nlm.nih.gov/pubmed/26723483
42. Choi YS, Kim C, Moon JH, Lee JY. Removal and killing of multispecies endodontic biofilms by $\mathrm{N}$-acetylcysteine. Braz J Microbiol. 2018; 49: 184-188.

PubMed: https://www.ncbi.nlm.nih.gov/pubmed/28916389

43. Dinicola S, De Grazia S, Carlomagno G, Pintucci JP. N-acetylcysteine as powerful molecule to destroy bacterial biofilms. A systematic review. Eur Rev Med Pharmacol Sci. 2014; 18: 2942-2948.

PubMed: https://www.ncbi.nlm.nih.gov/pubmed/25339490

44. Ham Y, Kim TJ. Inhibitory activity of monoacylglycerols on biofilm formation in Aeromonas hydrophila, Streptococcus mutans, Xanthomonas oryzae, and Yersinia enterocolitica. Springerplus. 2016; 5: 1526. PubMed: https://www.ncbi.nlm.nih.gov/pubmed/27652099

45. Richter K, Facal P, Thomas N, Vandecandelaere I, Ramezanpour $\mathrm{M}$, et al. Taking the Silver Bullet Colloidal Silver Particles for the Topical Treatment of Biofilm-Related Infections; Taking the Silver Bullet Colloidal Silver Particles for the Topical Treatment of BiofilmRelated Infections. ACS Appl Mater Interfaces. 2017; 9: 21631-21638. PubMed: https://www.ncbi.nlm.nih.gov/pubmed/28598149

46. Gujie Mi, Di Shi, Mian Wang, Thomas J. Webster Adv. Reducing Bacterial Infections and Biofilm Formation Using Nanoparticles and Nanostructured Antibacterial Surfaces. Healthcare Mater. 2018; 7.

47. Sousa C, Botelho C, Oliveira R. Nanotechnology applied to medical biofilms control. communicating current research and technological advances A. Méndez-Vilas (Ed.). 2011.

48. Soto SM. Biofilms in Urinary Tract Infections: New Therapeutic Approaches. Advances in Biology. 2014.

49. Braj RS, Brahma NS, Akanksha S, Wasi Khan, Alim HN, et al. Mycofabricated biosilver nanoparticles interrupt Pseudomonas aeruginosa quorum sensing systems. Sci Rep. 2015; 5: 13719. PubMed: https://www.ncbi.nlm.nih.gov/pubmed/26347993

50. Kaiser SJ, Mutters NT, Blessing B, Günther F. Natural isothiocyanates express antimicrobial activity against developing and mature biofilms of Pseudomonas aeruginosa. Fitoterapia. 2017; 119: 57-63. PubMed: https://www.ncbi.nlm.nih.gov/pubmed/28390975

51. Fu B, Wu Q, Dang M, Bai D, Guo Q, et al. Inhibition of Pseudomonas aeruginosa Biofilm Formation by Traditional Chinese Medicinal Herb Herba patriniae. Biomed Res Int. 2017; 2017: 9584703. PubMed: https://www.ncbi.nlm.nih.gov/pubmed/28377931

52. Kumar L, Chhibber S, Kumar R, Kumar M, Harjai K. Zingerone silences quorum sensing and attenuates virulence of Pseudomonas aeruginosa. Fitoterapia. 2015; 102: 84-95.

PubMed: https://www.ncbi.nlm.nih.gov/pubmed/25704369

53. Farias KS, Kato NN, Boaretto AG, Weber Jl, Brust FR, et al. Nectandra as a renewable source for (+)-?-bisabolol, an antibiofilm and antiTrichomonas vaginalis compound. Fitoterapia. 2019; 136: 104179. PubMed: https://www.ncbi.nlm.nih.gov/pubmed/31121252

54. Tse-Kai Fu, Sim-Kun Ng, Yi-En Chen, Yuan-Chuan Lee, Fruzsina Demeter, et al. Rhamnose Binding Protein as an Anti-Bacterial AgentTargeting Biofilm of Pseudomonas aeruginosa. Mar Drugs. 2019; 17. PubMed: https://www.ncbi.nlm.nih.gov/pubmed/31207891

55. Lokender K, Christopher RC, Susanta KM. Metalloprotease-1 inhibits and disrupts Enterococcus faecalis biofilms. Sarkar PLoS One. 2019; 14.

56. Yucui L, Yanjie Xu, Qiuhang S, Fei W, Luguo S, et al. Anti-biofilm Activities from Bergenia crassifolia Leaves against Streptococcus mutans. Front Microbiol. 2017; 8: 1738.

PubMed: https://www.ncbi.nlm.nih.gov/pmc/articles/PMC5601420

57. Deep G, Panigrahi GK. Hypoxia-Induced Signaling Promotes Prostate CancerProgression:ExosomesRoleas Messenger of HypoxicResponse 
in Tumor Microenvironment. Crit Rev Oncog. 2015; 20: 419-434. PubMed: https://www.ncbi.nlm.nih.gov/pubmed/27279239

58. Massimo A, Shabnam S, Youngjin K, Christina AM Jamieson. Tissue injury and hypoxia promote malignant progression of prostate cancer by inducing CXCL13 expression in tumor myofibroblasts, and Michael Karin. PNAS. 2014; 111: 14776-14781.

PubMed: https://www.ncbi.nlm.nih.gov/pmc/articles/PMC4205637

59. Gustafsson MV, Zheng X, Pereira T, Gradin K, Jin S, et al. Hypoxia requires notch signaling to maintain the undifferentiated cell state. Dev Cell. 2005; 9: 617-628.

PubMed: https://www.ncbi.nlm.nih.gov/pubmed/16256737

60. Cao Y, Ma J. Body mass index, prostate cancer-specific mortality, and biochemical recurrence: a systematic review and meta-analysis.
Cancer Prev Res (Phila). 2011; 4: 486-450.

PubMed: https://www.ncbi.nlm.nih.gov/pubmed/21233290

61. Stark T, Livas L, Natasha K. Inflammation in prostatecancer progression and therapeutic targeting. Transl Androl Urol. 2015; 4: 455-463. PubMed: https://www.ncbi.nlm.nih.gov/pubmed/26816843

62. Danielle SB, Hyun Koo. Targeted, triggered drug delivery to tumor and biofilm micro environment. Nano Medicine (Lond). 2016; 11: 873-879. PubMed: https://www.ncbi.nlm.nih.gov/pubmed/26987892

63. Guillaume L, Luis Estévez-Salmeron, Steve Oh, David L, Beverly ME, et al. An analogy between the evolution of drug resistance in bacterial communities and malignant tissues. NatRev Cancer. 2011;11:375-382. PubMed: https://www.ncbi.nlm.nih.gov/pubmed/21508974

64. Kresser. How to Treat Biofilms. 2018 